\title{
Molecular Gas Contents and Scaling Relations for Massive, Passive Galaxies at Intermediate Redshifts from the LEGA-C Survey
}

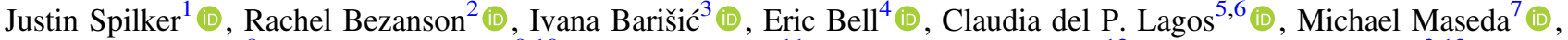 \\ Adam Muzzin $^{8}$ (D), Camilla Pacifici ${ }^{9,10}$ (D), David Sobral ${ }^{11}$ (D), Caroline Straatman ${ }^{12}$ (D), Arjen van der Wel ${ }^{3,12}$ (iD), \\ Pieter van Dokkum ${ }^{13}$ (D), Benjamin Weiner ${ }^{14}$ (D), Katherine Whitaker ${ }^{15}$ (D), Christina C. Williams ${ }^{14,16}$ (D), and Po-Feng Wu ${ }^{3}$ (D) \\ ${ }^{1}$ Department of Astronomy, University of Texas at Austin, 2515 Speedway, Stop C1400, Austin, TX 78712, USA; spilkerj@gmail.com \\ ${ }^{2}$ Department of Physics and Astronomy and PITT PACC, University of Pittsburgh, Pittsburgh, PA 15260, USA \\ ${ }^{3}$ Max-Planck-Institut für Astronomie, Königstuhl 17, D-69117, Heidelberg, Germany \\ ${ }^{4}$ Department of Astronomy, University of Michigan, 1085 South University Avenue, Ann Arbor, MI 48109-1107, USA \\ ${ }^{5}$ International Centre for Radio Astronomy Research, M468, University of Western Australia, 35 Stirling Hwy, Crawley, WA 6009, Australia \\ ${ }^{6}$ ARC Centre of Excellence for All Sky Astrophysics in 3 Dimensions (ASTRO 3D), 44 Rosehill Street Redfern, NSW 2016, Australia \\ ${ }^{7}$ Leiden Observatory, Leiden University, P.O. Box 9513, 2300 RA Leiden, The Netherlands \\ ${ }^{8}$ Department of Physics and Astronomy, York University, 4700 Keele St., Toronto, Ontario, M3J 1P3, Canada \\ Astrophysics Science Division, Goddard Space Flight Center, Code 665, Greenbelt, MD 20771, USA \\ ${ }^{10}$ Space Telescope Science Institute, 3700 San Martin Drive, Baltimore, MD 21218, USA \\ ${ }_{11}$ Physics Department, Lancaster University, Lancaster LA1 4 YB, UK \\ ${ }^{12}$ Sterrenkundig Observatorium, Universiteit Gent, Krijgslaan 281 S9, B-9000 Gent, Belgium \\ ${ }_{13}$ Astronomy Department, Yale University, New Haven, CT 06511, USA \\ ${ }^{14}$ Steward Observatory, University of Arizona, 933 North Cherry Avenue, Tucson, AZ 85721, USA \\ ${ }^{15}$ Department of Physics, University of Connecticut, 2152 Hillside Road, Unit 3046, Storrs, CT 06269, USA \\ Received 2018 April 13; accepted 2018 May 4; published 2018 June 18
}

\begin{abstract}
A decade of study has established that the molecular gas properties of star-forming galaxies follow coherent scaling relations out to $z \sim 3$, suggesting remarkable regularity of the interplay between molecular gas, star formation, and stellar growth. Passive galaxies, however, are expected to be gas-poor and therefore faint, and thus little is known about molecular gas in passive galaxies beyond the local universe. Here we present deep Atacama Large Millimeter/submillimeter Array observations of $\mathrm{CO}(2-1)$ emission in eight massive $\left(M_{\text {star }} \sim 10^{11} M_{\odot}\right)$ galaxies at $z \sim 0.7$ selected to lie a factor of 3-10 below the star-forming sequence at this redshift, drawn from the Large Early Galaxy Astrophysics Census survey. We significantly detect half the sample, finding molecular gas fractions $\lesssim 0.1$. We show that the molecular and stellar rotational axes are broadly consistent, arguing that the molecular gas was not accreted after the galaxies became quiescent. We find that scaling relations extrapolated from the star-forming population overpredict both the gas fraction and gas depletion time for passive objects, suggesting the existence of either a break or large increase in scatter in these relations at low specific star formation rate. Finally, we show that the gas fractions of the passive galaxies we have observed at intermediate redshifts are naturally consistent with evolution into local, massive early-type galaxies by continued low-level star formation, with no need for further gas accretion or dynamical stabilization of the gas reservoirs in the intervening 6 billion years.
\end{abstract}

Key words: galaxies: evolution - galaxies: high-redshift - galaxies: ISM

\section{Introduction}

The processes by which galaxies grow and evolve are intimately linked to the accretion and conversion of gas into stars. In particular, because stars form from molecular gas (e.g., Schruba et al. 2011), the heating, cooling, and transport of gas from outside and within galaxies play a large role in determining how efficiently a galaxy can form stars and the overall mass of stars that can be formed. By and large, galaxies form stars across cosmic time in equilibrium with the supply of fresh gas from accretion and mergers; the interplay between gas accretion, outflows, star formation, and mergers naturally regulates the growth of galaxies (e.g., Davé et al. 2011, 2012; Lilly et al. 2013; Peng \& Maiolino 2014).

Surveys of increasingly large numbers of galaxies have shown that the majority of galaxies exhibit a relatively tight and nearly linear relationship between the current star formation rate (SFR) and the mass of stars already formed ( $M_{\text {star }}$; e.g., Noeske et al. 2007; Franx et al. 2008; Whitaker et al. 2012,

\footnotetext{
${ }^{16}$ NSF Fellow.
}

2014; Speagle et al. 2014; Schreiber et al. 2016). The intrinsic scatter in the relationship is $\approx 0.3 \mathrm{dex}$, and objects that lie near it are generally considered to be "normal" galaxies. The normalization of the "star-forming sequence" increases rapidly with redshift, implying much more rapid galaxy growth in the early universe as compared to today, or an overall increase in the SFR density function (Sobral et al. 2014). At the most massive end, however, an ever-larger fraction of galaxies exhibit markedly depressed SFRs (or specific SFR, $\left.\mathrm{sSFR} \equiv \mathrm{SFR} / M_{\text {star }}\right)$. This transition occurs near the break in the stellar mass function, $\log M_{\text {star }} / M_{\odot} \gtrsim 10.5-11$, and does not appear to evolve significantly with redshift (e.g., Peng et al. 2010, though see also Gavazzi et al. 2015; Tomczak et al. 2016). Star formation in these massive galaxies appears to be efficiently shut off ("quenched") as they transition to the red sequence, but the physical mechanisms responsible for this quenching are still unclear.

After a decade of extensive observational investment, the relationship between gas supply and the growth of star-forming galaxies has become more clear, both at low redshift (e.g., Saintonge et al. 2011, 2017; Bothwell et al. 2014) and in the 
distant universe (e.g., Tacconi et al. 2013; Magnelli et al. 2014; Papovich et al. 2016; Scoville et al. 2016). For galaxies with SFRs near and above the star-forming sequence, scaling relations have been derived relating the properties of the molecular interstellar medium (ISM) with other galaxy properties (e.g., Genzel et al. 2015; Scoville et al. 2017; Tacconi et al. 2018), with remarkably good agreement between various tracers of the molecular gas. In general, the molecular gas fraction $f_{\mathrm{H}_{2}} \equiv M_{\mathrm{H}_{2}} / M_{\text {star }}$ increases rapidly with redshift, is elevated for galaxies well above the star-forming sequence, and shows a weak decline with increasing $M_{\text {star }}$. The gas depletion time, $t_{\mathrm{dep}} \equiv M_{\mathrm{H}_{2}} / \mathrm{SFR}$, a measure of how long a galaxy could continue to form stars at its current rate before exhausting its gas supply, shows a much weaker evolution with redshift, is shorter for galaxies above the star-forming sequence, and is either constant or weakly increases toward high $M_{\text {star }}$. Typical values for Milky Way-mass star-forming galaxies at $z \sim 0$ are $f_{\mathrm{H}_{2}} \sim$ few $\times 10^{-2}$ and $t_{\mathrm{dep}} \sim 1 \mathrm{Gyr}$, and at $z \sim 1$, $f_{\mathrm{H}_{2}} \sim 0.5-0.8$ and $t_{\text {dep }} \sim 0.7$ Gyr (Tacconi et al. 2018).

The recent advent of large samples of "normal" star-forming galaxies with molecular gas measurements has been particularly useful at high redshifts, providing extremely valuable reference samples for other galaxies that may be less "normal." Of particular relevance for the buildup of the quiescent galaxy population, molecular gas observations have now also targeted smaller samples of galaxies thought to be actively quenching star formation for various reasons (e.g., Geach et al. 2013; Spilker et al. 2016; Popping et al. 2017) or that show spectral signatures of quenching in the past $<1$ Gyr (Suess et al. 2017). These studies indicate that, while the suppression of star formation does not require the complete removal or depletion of the molecular gas, the quenching processes do appear to lower $f_{\mathrm{H}_{2}}$ at fixed mass compared to the reference samples in most cases.

Because of the relationship between $M_{\mathrm{H}_{2}}$ and SFR, however, much less is known about the molecular ISM in galaxies well below the star-forming sequence; the expected molecular masses require very sensitive observations and substantial integration times even with the supreme sensitivity of the Atacama Large Millimeter/submillimeter Array (ALMA). Observations of local massive and passive early-type galaxies, for example, reveal gas fractions an imposing 1-2 orders of magnitude lower than their star-forming counterparts (Young et al. 2011; Davis et al. 2013, 2016). Because these local galaxies show few signs of recent star formation for the past many Gyr, however, it is not clear to what extent inferences about the suppression of star formation in these galaxies also apply in the distant universe, closer to the epoch at which galaxies first became quiescent.

The main focus of our work here is to determine whether the scaling relations derived from observations of star-forming galaxies and cosmological simulations can accurately predict or be extrapolated down to passive galaxies at intermediate redshift. We present ALMA observations of a sample of eight massive $\left(\log M_{\text {star }} / M_{\odot}>10.8\right)$ galaxies at $z \sim 0.7$ from the Large Early Galaxy Astrophysical Census (LEGA-C) survey selected to lie a factor of 3-10 below the star-forming sequence at this redshift. We observed the $\mathrm{CO}(2-1)$ transition, a tracer of the molecular ISM. In Section 2, we describe the parent LEGA-C sample, our ALMA observations, and our measurements of $M_{\mathrm{H}_{2}}$. Section 3 provides a broad overview of our basic results, and we compare the stellar and molecular dynamics of our detected galaxies. In Section 4, we compare the gas fractions and depletion times derived for our sample with observationally based scaling relations and with the EAGLE cosmological simulation. We discuss the implications our observations have for the understanding of galaxy quenching in Section 5, and we conclude in Section 6. Throughout, we assume a flat $\Lambda$ CDM cosmology with $\Omega_{m}=0.307$ and $H_{0}=67.7 \mathrm{~km} \mathrm{~s}^{-1} \mathrm{Mpc}^{-1}$ (Planck Collaboration et al. 2016).

\section{Data and Analysis}

\subsection{LEGA-C and Selection of Quiescent Galaxies}

We selected galaxies for $\mathrm{CO}(2-1)$ observations from the LEGA-C survey of $0.6<z<1.0$ galaxies. The survey is described in detail by van der Wel et al. (2016). Briefly, galaxies were selected based on $K$-band magnitude from the UltraVISTA catalog described in Muzzin et al. (2013). The LEGA-C survey consists of $\sim 3200$ galaxies observed with 20 $\mathrm{hr}$ integrations using the VIMOS spectrograph on the Very Large Telescope. The LEGA-C spectra yield high signal-tonoise detections of the stellar absorption features and continuum, allowing determinations of the age of the stellar populations, metallicities, and stellar velocity dispersions. Because the LEGA-C survey targets the COSMOS extragalactic survey field, full panchromatic spectral energy distribution (SED) information is available, along with morphological information from Hubble Space Telescope observations. The galaxies studied in this work were selected from the first data release (DR1) catalog for which VIMOS observations were completed by early 2016, consisting of 644 objects in the primary sample with good spectra. This early sample represents a subset of the full survey area and is not biased with respect to the full LEGA-C sample in terms of, for example, $K$-band magnitude. Note that, while the galaxies were selected from the initial DR1 catalog, the figures and table in this work use values from the updated DR2 catalog (1989 galaxies; C. Straatman et al. 2018, in preparation). Stellar masses of the LEGA-C sample have been measured by fitting the photometric SED using FAST (Kriek et al. 2009) assuming a Chabrier (2003) initial mass function.

\subsubsection{SFR Estimation}

The primary SFR estimates for the LEGA-C sample come from modeling of the SED of our target galaxies, from the ultraviolet to the mid-infrared. SFRs include the unobscured and obscured components based on UV and IR $(24 \mu \mathrm{m})$ fluxes. The low resolution of the Spitzer/MIPS $24 \mu \mathrm{m}$ imaging requires the use of a deblending procedure to assign the measured $24 \mu \mathrm{m}$ flux to $K$-band-detected galaxies (Muzzin et al. 2013). In our final ALMA sample, described further below, two objects (IDs 130284 and 132776) are near bright $24 \mu \mathrm{m}$ sources, where the deblending is potentially unreliable. For all objects in the ALMA sample, the inclusion of the $24 \mu \mathrm{m}$ fluxes increases the inferred SFR by a factor of 2.5 , on average. Unsurprisingly, none of these objects with low SFR are detected in Herschel Space Observatory imaging of the COSMOS field.

Several authors have noted that the IR luminosity (or observed-frame $24 \mu \mathrm{m}$ luminosity, generally the only available tracer of dust emission at low SFR and high redshift) can overestimate the obscured SFR of quiescent galaxies, in some cases quite severely (e.g., Salim et al. 2009; 
Table 1

ALMA-observed LEGA-C Sample Target Properties

\begin{tabular}{|c|c|c|c|c|c|c|c|c|}
\hline $\begin{array}{c}\text { LEGA-C ID } \\
\ldots\end{array}$ & $\begin{array}{c}\text { R.A. } \\
\ldots\end{array}$ & $\begin{array}{c}\text { Decl. } \\
\ldots\end{array}$ & $\begin{array}{c}z_{\text {spec }} \\
\cdots\end{array}$ & $\begin{array}{c}\log M_{\text {star }} / M_{\odot} \\
\ldots\end{array}$ & $\begin{array}{c}\mathrm{SFR} \\
M_{\odot} \mathrm{yr}^{-1}\end{array}$ & $\begin{array}{l}\sigma_{100 \mathrm{~km} \mathrm{~s}^{-1}}{ }^{\mathrm{a}} \\
\mu \mathrm{Jy} / \text { beam }\end{array}$ & $\begin{array}{c}S_{\mathrm{CO}(2-1)} \Delta v \\
\mathrm{Jy} \mathrm{km} \mathrm{s}^{-1}\end{array}$ & $\begin{array}{c}\log M_{\mathrm{H}_{2}} / M_{\odot} \\
\ldots\end{array}$ \\
\hline 74512 & $10^{\mathrm{h}} 01^{\mathrm{m}} 42^{\mathrm{s}} .88$ & $+02^{\circ} 01^{\prime} 21^{\prime \prime} .9$ & 0.7330 & 10.96 & 6.3 & 132 & $0.16 \pm 0.04$ & $9.82 \pm 0.13$ \\
\hline 130284 & $10^{\mathrm{h}} 00^{\mathrm{m}} 13^{\mathrm{s}} .78$ & $+02^{\circ} 19^{\prime} 37^{\prime \prime} 0$ & 0.6017 & 10.96 & 6.8 & 151 & $0.36 \pm 0.04$ & $10.00 \pm 0.06$ \\
\hline 132776 & $10^{\mathrm{h}} 00^{\mathrm{m}} 12^{\mathrm{s}} .43$ & $+02^{\circ} 21^{\prime} 21^{\prime \prime} 9$ & 0.7500 & 10.98 & 7.9 & 163 & $0.33 \pm 0.07$ & $10.16 \pm 0.11$ \\
\hline 138718 & $10^{\mathrm{h}} 00^{\mathrm{m}} 13^{\mathrm{s}} .89$ & $+02^{\circ} 25^{\prime} 38^{\prime \prime} 0$ & 0.6558 & 11.13 & 5.6 & 188 & $<0.21$ & $<9.84$ \\
\hline 169076 & $09^{\mathrm{h}} 59^{\mathrm{m}} 07^{\mathrm{s}} .30$ & $+02^{\circ} 19^{\prime} 05^{\prime \prime} 8$ & 0.6772 & 11.49 & 5.1 & 256 & $<0.23$ & $<9.91$ \\
\hline & Stacked nondetections & & 0.6754 & 11.31 & 5.3 & 106 & $<0.093$ & $<9.51$ \\
\hline
\end{tabular}

Note. LEGA-C ID numbers are the same as in the UltraVISTA catalog of Muzzin et al. (2013). Stellar masses are determined by fitting to multiwavelength photometry using FAST. SFRs are based on a weighted sum of UV and IR $(24 \mu \mathrm{m})$ fluxes. Integrated $\mathrm{CO}(2-1)$ line fluxes are converted to molecular gas masses under the assumptions described in Section 2.3. Upper limits for nondetections are $3 \sigma$, and molecular gas masses can be rescaled under different assumptions as $M_{\mathrm{H}_{2}}\left(0.8 / r_{21}\right)\left(\alpha_{\mathrm{CO}} / 4.4\right)$.

${ }^{a}$ ALMA rms sensitivity in $100 \mathrm{~km} \mathrm{~s}^{-2}$ channels, naturally weighted images.

Hayward et al. 2014; Utomo et al. 2014; Man et al. 2016). This can be due to several effects, including the fact that the IR luminosity is a long-lived tracer compared to the instantaneous SFR, and $24 \mu \mathrm{m}$ emission can be boosted by dust heating unrelated to star formation, including circumstellar dust heated by intermediate-age asymptotic giant branch (AGB) stars, extended cirrus dust heated by old stellar populations, or weak nuclear activity. The latter three result in a higher $L_{\mathrm{IR}} / \mathrm{SFR}$ ratio, or equivalently only a fraction of the observed $L_{\mathrm{IR}}$ should be considered in the calculation of the SFR.

Of these options, weak nuclear activity is unlikely, with the $24 \mu \mathrm{m}$ emission expected from stacking of X-ray images falling 3 orders of magnitude below the observed emission for intermediate-redshift quiescent galaxies (Fumagalli et al. 2014). Dust heating from AGB stars and cirrus dust are potentially more relevant. Fumagalli et al. used a stacking analysis of MIPS $/ 24 \mu \mathrm{m}$ images of quiescent galaxies at $0.3<z<2.5$ and found that the SFR determined from these images could be overestimated by an order of magnitude. However, there is reason to believe the situation is not so dire for our own selection. Fumagalli et al. focused on galaxies much more quiescent than ours, $20-40 \times$ below the starforming sequence, compared to our own selection of $3-10 \times$ below. The objects in our sample do not reach such low sSFR even if none of the IR emission is related to star formation and only the SFR based on the rest-UV is considered. The SFR overestimation becomes less severe at higher sSFR because true obscured star formation rapidly outshines the lower-level IR emission unrelated to SF. For the sSFRs typical of our sample, the work by Fumagalli et al. (2014) indicates that the SFRs should be overestimated by less than a factor of $\sim 2$.

\subsubsection{ALMA Selection}

We selected galaxies from the LEGA-C sample with spectroscopic redshift $z<0.84$, for which the $\mathrm{CO}(2-1)$ line is observable with the ALMA Band 4 receivers. We selected massive galaxies, with $\log M_{\text {star }} / M_{\odot}>10.8$ and SFR 3-10× below the $z \sim 0.7$ star-forming sequence as defined by Whitaker et al. (2012). Given the intrinsic scatter in this sequence $(\approx 0.3 \mathrm{dex})$, this selects objects with SFRs lower than $\approx 90 \%$ of all galaxies at this redshift. We further required that
SFR $>2.5 M_{\odot} \mathrm{yr}^{-1}$, intended to restrict the sample to galaxies that could plausibly be detected in $\mathrm{CO}$ in a reasonable integration time with ALMA. This resulted in a sample of 65 galaxies, from which we selected eight for observation with ALMA, listed in Table 1. We aimed to include galaxies with a variety of morphologies, both obvious spiral galaxies and bulge-dominated elliptical galaxies. Two of the eight galaxies (IDs 110509 and 169076) are identified as radio-loud active galactic nuclei (AGNs; Barišić et al. 2017); both lack optical emission lines and are classified as low-excitation radio galaxies. The sensitivity of the best-available radio imaging of the COSMOS field (Smolčić et al. 2017) is not sufficient to detect radio continuum emission related to star formation for our sample (approximate $3 \sigma$ limiting SFR $>18 M_{\odot} \mathrm{yr}^{-1}$ ). The VIMOS spectra of our eight galaxies are shown in Figure 1.

For two objects in the ALMA sample, the LEGA-C spectra clearly detect the $[\mathrm{O}$ II $] \lambda 3727$ doublet, which can also be used as an SFR indicator, although one highly susceptible to dust extinction and low-level nuclear activity. Converting this line flux to an SFR yields values less than the UV+IR SFRs, as expected, but within $\sim 2 \times$ the UV-only SFR. The remaining objects show little evidence for nebular emission lines also used as SFR indicators (e.g., $\mathrm{H} \beta$ ), with any emission generally overwhelmed by the strong absorption features.

In Figure 2 we show the selection of the ALMA sample in the SFR- $M_{\text {star }}$ plane with respect to the full LEGA-C sample and all other LEGA-C objects that meet our selection criteria. We also show the size-mass relation for star-forming and quiescent galaxies, with the best-fit relations determined by van der Wel et al. (2014). Because we chose objects with a range of morphologies, it is unsurprising that the ALMA sample includes objects that both follow and do not follow the expected size-mass relation for quiescent galaxies.

Figure 3 shows two other relevant diagrams that give the ALMA sample additional context. The top panel shows the ALMA sample in $U V J$ color-color space, with the division between star-forming and quiescent objects from Muzzin et al. (2013) also shown. The boundary between these two populations in $U V J$ colors is not a definitive one, with varying definitions found in the literature, as well as an expectation that galaxies must transition from one region to the other over time. Although this diagram was not used in the selection of the ALMA sample, it is reassuring that six of the eight targets lie 

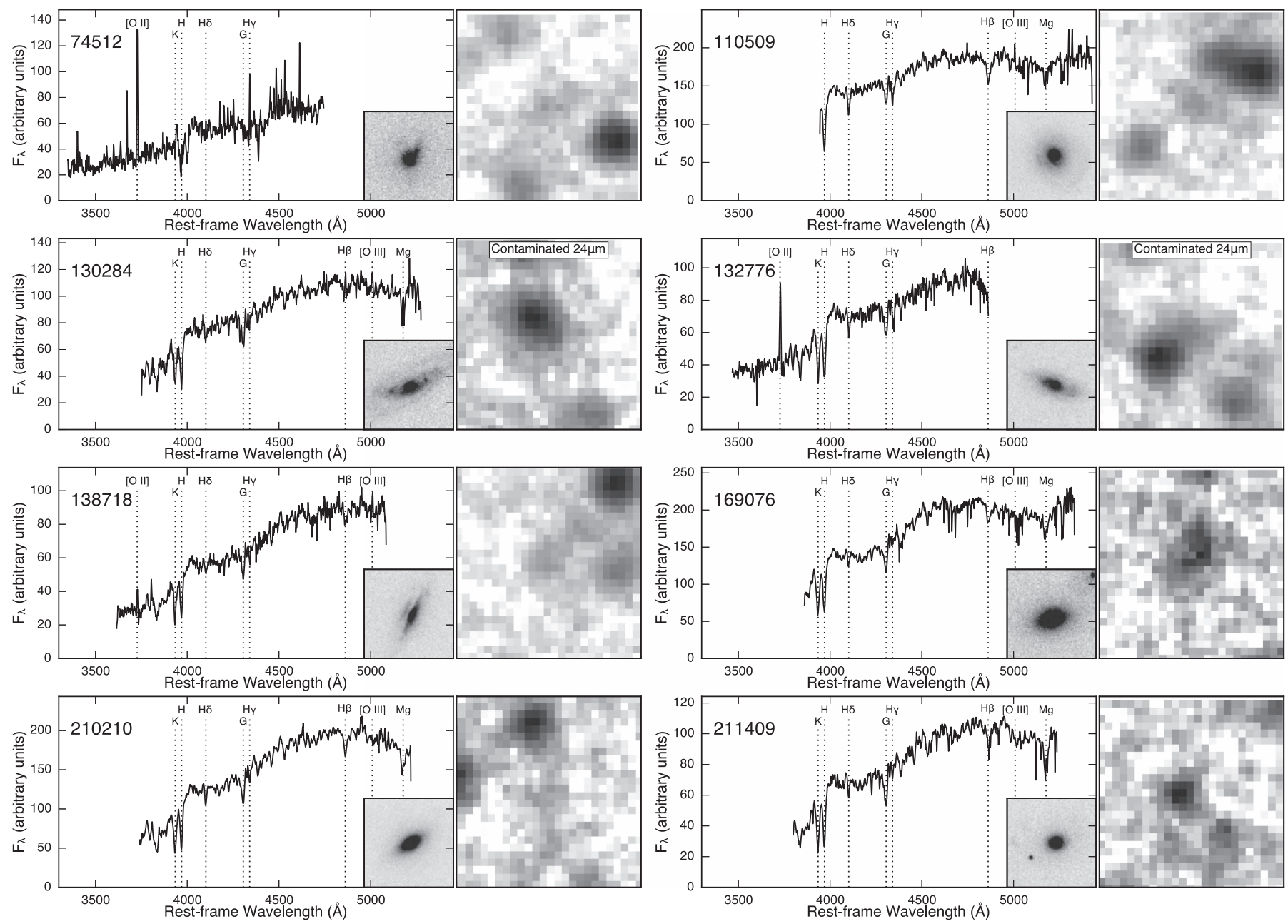

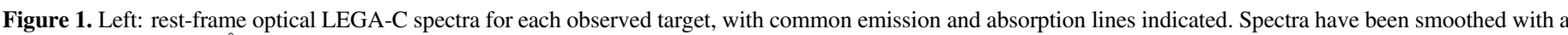

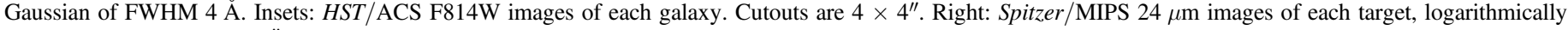

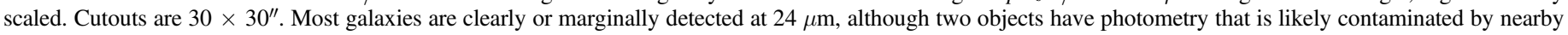
galaxies.

within (given the uncertainties) the region of the $U V J$ color-color diagram generally occupied by quiescent galaxies. Of the two remaining objects, one (ID 74512) lies in the region generally occupied by star-forming galaxies; the other (ID 138718) lies in the region generally occupied by dusty galaxies.

The lower panel of Figure 3 shows the distribution of the $D_{n} 4000$ index against the equivalent width of $\mathrm{H} \delta$ for the LEGA-C and ALMA-observed samples (Wu et al. 2018; an emission line template has been subtracted from the spectra). This diagram is useful as a proxy for the stellar age, older galaxies showing lower $\mathrm{H} \delta$ equivalent width and higher $D_{n} 4000$. These quantities are also correlated with $M_{\text {star }}$, indicating older stellar populations in massive galaxies (e.g., Kauffmann et al. 2003; Maltby et al. 2016). Based on this diagram, we infer typical stellar ages for the ALMA-observed galaxies of 1-3 Gyr, depending on the assumed star formation history; a similar result is obtained through fitting to the full LEGA-C spectra (Chauke et al. 2018). In other words, the objects we observed with ALMA have been passive for quite some time. They show little evidence for significant recent star formation, unlike galaxies selected with "poststarburst" criteria that emphasize the recent or impending quenching of star formation (e.g., Tremonti et al. 2007; Sell et al. 2014; Suess et al. 2017).
Finally, we examined the local galaxy environments of our target objects using the estimates of Darvish et al. (2016). These estimates use photometric redshifts because the spectroscopic completeness in the COSMOS field is low. Darvish et al. note that at $z<1$, a comparison of spectroscopic and photometric redshifts shows that the redshift uncertainties are generally small, with a dispersion $\sigma_{z} \sim 0.008$, sufficiently small that the use of photometric redshifts does not wash out line-of-sight galaxy structures. For the LEGA-C sample as a whole, the median overdensity is $\log (1+\delta)=0.13$, while for the ALMA-observed sources, the median is 0.16 , with large uncertainties on both values. The ALMA targets are not in systematically overdense environments compared to the full LEGA-C sample or the field as a whole.

\subsection{ALMA Observations}

Basic details of our ALMA observations and target galaxies are summarized in Table 1. ALMA observations of our sample of eight galaxies were carried out in project 2016.1.00790.S (PI: Spilker) in separate observing sessions from 2017 January 17 to March 12 using the Band $4(2 \mathrm{~mm})$ receivers (Asayama et al. 2014). All observations were conducted with the array in a compact configuration, with $\sim 40$ antennas separated by 


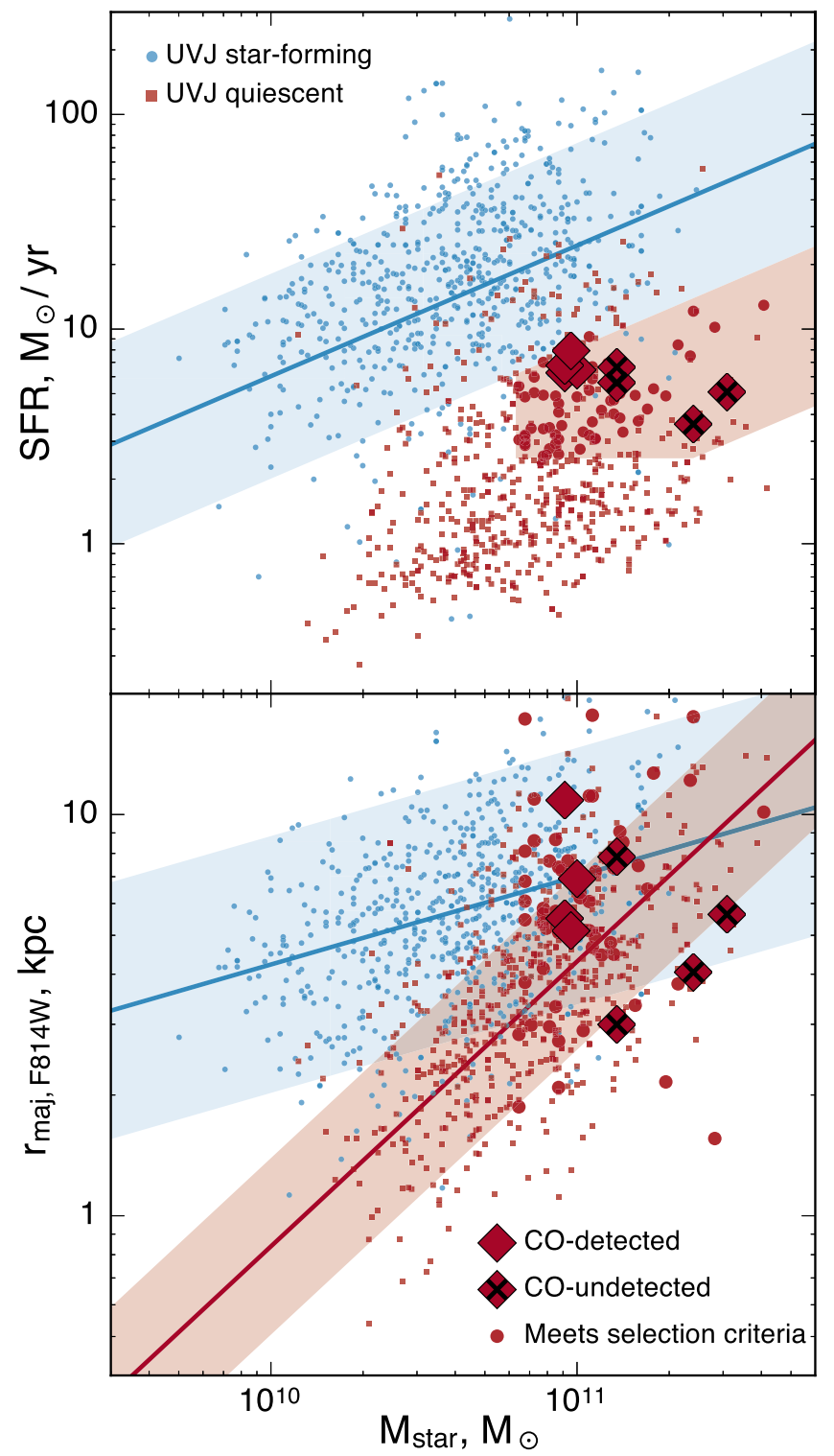

Figure 2. Selection of the ALMA-observed massive, passive sample with respect to the full LEGA-C sample. In both panels, the LEGA-C sample galaxies are color coded blue (red) if they are classified as star-forming (quiescent) in rest-frame $U V J$ space (see Figure 3). Red circles are all LEGA-C galaxies that meet our selection criteria, while the ALMA-observed objects are shown with large red diamonds; CO-undetected objects are also marked with a black $\times$. Top: The ALMA sample was primarily selected based on stellar mass and SFR; see text for details. The blue line shows the star-forming sequence at $z=0.7$ from Whitaker et al. (2012), and the blue shaded region encompasses SFRs a factor of 3 above and below the relation. The red shaded region shows our selection box of massive and passive galaxies. Bottom: size-mass relation for the LEGA-C and ALMA samples. Blue and red lines and regions show the size-mass relations for star-forming and quiescent galaxies at $z=0.75$ from van der Wel et al. (2014).

maximum baselines ranging from 272 to $330 \mathrm{~m}$. Observing sessions varied between 57 and 80 minutes in duration, with 37-49 minutes spent on-source per target. The precipitable water vapor levels varied between 2.7 and $4.5 \mathrm{~mm}$, resulting in typical system temperatures of 70-95 K. Quasars J1058+0133 or J0854+2006 served as bandpass calibrators, while the quasar J0948+0022 was observed for complex gain calibration for all sources. The absolute flux scale was determined using observations of Ganymede or one of J0854+2006 or J1058 +0133 , both of which are monitored regularly by ALMA.
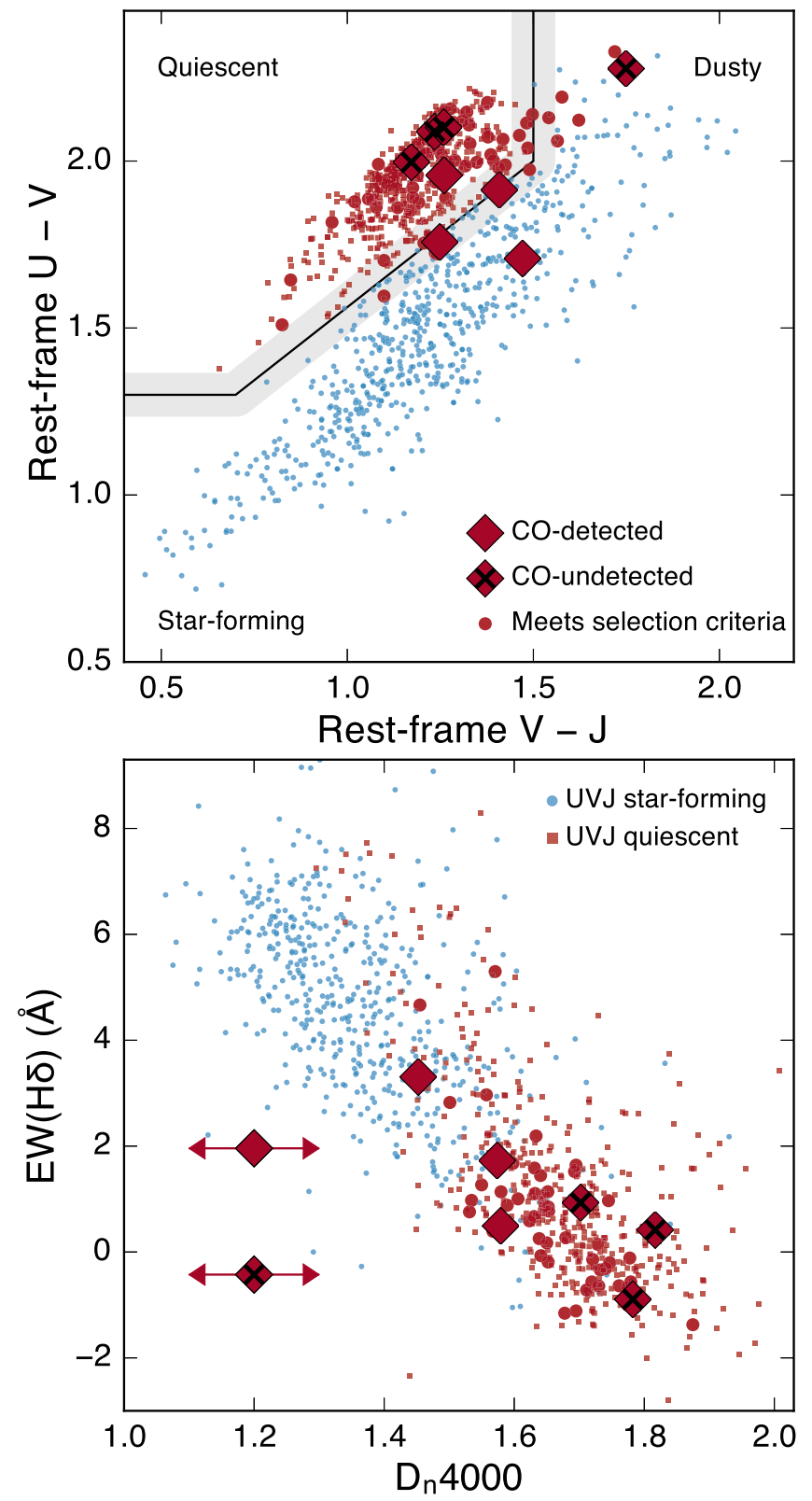

Figure 3. Symbols are plotted as in Figure 2. Top: rest-frame UVJ color-color diagram for the LEGA-C galaxies and the ALMA sample, with the division between star-forming and quiescent galaxies of Muzzin et al. (2013) shown with the black line. The gray shaded band around this line represents both the differences in divisions found in the literature and an expectation that galaxies must transition from one region to the other over a period of time. Although this diagram was not used in the selection of the ALMA sample, six of the eight targets lie within (given the uncertainties) the quiescent region of the diagram at upper left. Bottom: the $D_{n} 4000$ index against the $\mathrm{H} \delta$ equivalent width, a proxy for the age of the stellar populations, with older and more massive systems located toward the lower right. We infer typical stellar ages of 1-3 Gyr for the ALMA sample; these objects are not recently quenched. For two objects (IDs 110509 and 169076), the LEGA-C spectra do not extend sufficiently blueward to measure $D_{n} 4000$, shown arbitrarily at $D_{n} 4000=1.2$ with arrows in each direction.

The correlator was configured to observe the $\mathrm{CO}(2-1)$ line at the known redshift of each target with one baseband with $7.812 \mathrm{MHz}$ channelization $\left(\approx 16 \mathrm{~km} \mathrm{~s}^{-1}\right)$ after correlator preaveraging by a factor of 8 . Three further basebands with $1.875 \mathrm{GHz}$ usable bandwidth each were placed at higher frequencies for continuum observations. All data were reduced 

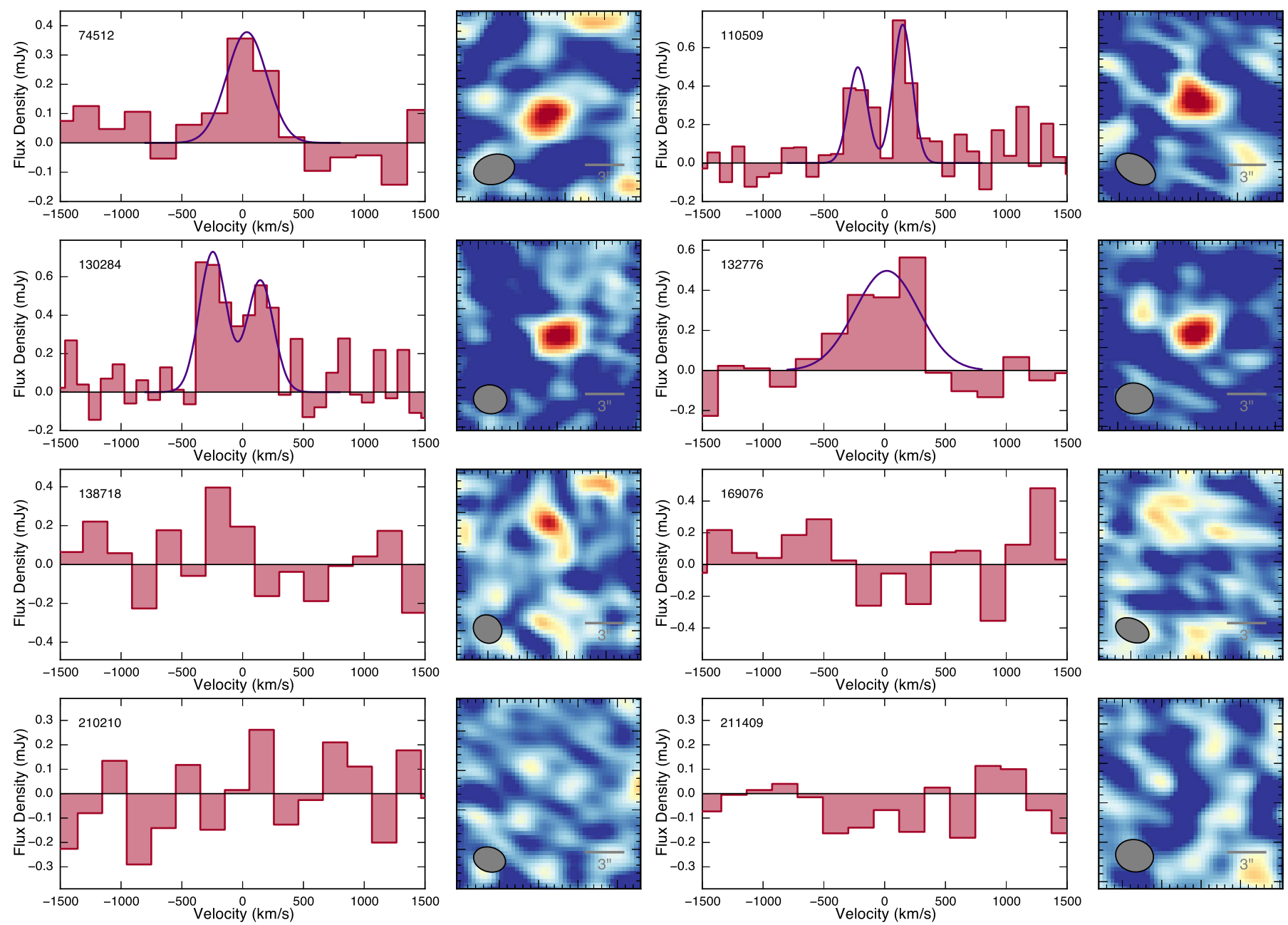

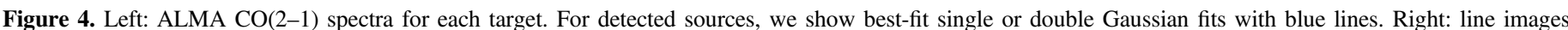

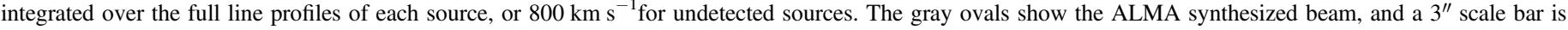
indicated.

using the standard ALMA pipeline, with manual inspection of the quality of the reduction.

For each object, we produce continuum images and $\mathrm{CO}(2-1)$ spectral cubes with various frequency channelization. All data were imaged with a natural weighting of the visibilities, which maximizes sensitivity to faint emission in exchange for slightly lower spatial resolution. The effective spatial resolution of the data imaged in this way ranges from 1 !" 9 to 2 !" 4 ( $\approx 13-17 \mathrm{kpc})$. No $2 \mathrm{~mm}$ continuum emission is detected in any target. We extract integrated spectra for all targets by fitting point-source models to the visibility data directly, averaging six or 12 channels to create spectra with velocity resolution $\sim 100-200 \mathrm{~km} \mathrm{~s}^{-1}$. When averaging over the full line profiles, we see evidence that most of the detected sources are marginally spatially resolved (e.g., by comparing the peak pixel values with spatially integrated fluxes), but this effect is negligible in narrower channels.

Finally, because all of our targets are located in the COSMOS extragalactic deep field, we checked the ALMA archive to determine if any other observations of our targets were publicly available or fell within the footprint of other projects. No other observations were found.

\subsection{Molecular Gas Masses}

Spectra and integrated line images of each target are shown in Figure 4. We clearly detect four of the eight targets in $\mathrm{CO}(2-1)$ emission. Two of the four detections show doublepeaked line profiles, usually indicative of rotating disks; the other two sources are centrally peaked. We discuss kinematics in more detail in Section 3.2. Integrated line fluxes are determined by fitting either one or two Gaussian profiles, also shown in Figure 4. For the undetected sources, we estimate upper limits on the $\mathrm{CO}$ emission by determining the noise in channels $800 \mathrm{~km} \mathrm{~s}^{-1}$ wide, which would fully encompass all of the line emission in all detected sources. The upper limits on the integrated line flux are proportional to $\sqrt{\Delta v}$, where $\Delta v$ is the velocity interval over which the spectrum is integrated, so using very wide channels results in conservative upper limits. The integrated line fluxes are given in Table 1.

We convert the observed CO luminosities and upper limits to estimates of the molecular gas masses under standard assumptions about the $\mathrm{CO}$ excitation and the $\mathrm{CO}-\mathrm{H}_{2}$ conversion factor $\alpha_{\mathrm{CO}}$. The effects of the unknown $\mathrm{CO}$ line excitation are minimal, as the $\mathrm{CO}(2-1)$ transition we have observed is very close to the ground-state $\mathrm{CO}(1-0)$ line typically used for 
molecular gas estimation. Observations of the Milky Way, nearby quiescent and star-forming galaxies, and high-redshift star-forming galaxies ubiquitously show that a line ratio $r_{21}=$ 0.7-1.0 (in brightness temperature units) encompasses the plausible expected range (Fixsen et al. 1999; Combes et al. 2007; Dannerbauer et al. 2009; Young et al. 2011; Spilker et al. 2014; Saintonge et al. 2017). In this work, we assume $r_{21}=0.8$.

The subsequent conversion between $\mathrm{CO}$ luminosity and molecular gas mass is also uncertain (for a recent review, see Bolatto et al. 2013). The conversion factor $\alpha_{\mathrm{CO}}$ is known to vary with the gas metallicity (e.g., Leroy et al. 2011), which affects the formation and destruction of $\mathrm{CO}$ molecules. The metallicities (either gas-phase or stellar) of our targets have not been measured, but are expected to be solar or near-solar based on the mass-metallicity relation. For example, Gallazzi et al. (2014) predict $\log Z / Z_{\odot} \sim 0.0-0.1$ for quiescent galaxies in our mass range, with a scatter of $\approx 0.2$. We therefore expect only minor variations in $\alpha_{\mathrm{CO}}$ due to metallicity effects. The $\mathrm{CO}-\mathrm{H}_{2}$ conversion factor also depends on the gas conditions and kinematics, which affect the optical depth of the CO transitions through radiative trapping. This is chiefly relevant for mergers and other high-SFR systems, in which increased gas turbulence or bulk motions lower the effective $\mathrm{CO}$ optical depth and also $\alpha_{\mathrm{CO}}$ (e.g., Narayanan et al. 2012; Spilker et al. 2015). These effects are also expected to be minor for our targeted objects, which have low SFRs, no signs of interaction, and evidence for disk-like rotation in many cases (see Section 3.2).

In this work, we adopt a "Milky Way-like" value, $\alpha_{\mathrm{CO}}=4.4 M_{\odot}\left(\mathrm{K} \mathrm{km} \mathrm{s}^{-1} \mathrm{pc}^{2}\right)^{-1}$ (e.g., Solomon et al. 1987; Bolatto et al. 2013; Sandstrom et al. 2013). This value agrees with standard dust-based methods we describe further as part of our stacking analysis below. While this choice is justified for the reasons already mentioned, it does still carry significant systematic uncertainty, likely of order $50 \%$. While this may result in adjustments to the absolute values of the gas masses we derive, the relative values are robust, and it does not affect the overall trends we find. Of relevance to our subsequent discussion in Section 4, the true gas masses of our sample are unlikely to be significantly larger than the values we infer. The molecular gas masses we derive can easily be rescaled using different assumptions, as $M_{\mathrm{H}_{2}}\left(0.8 / r_{21}\right)\left(\alpha_{\mathrm{CO}} / 4.4\right)$. When comparing to other samples and galaxies observed in $\mathrm{CO}$ by other authors, we also adjust their derived gas masses to match our adopted value of $\alpha_{\mathrm{CO}}$. As we are interested in normal starforming and quiescent galaxies, this adjustment is minor, no larger than $\sim 20 \%$ for the comparison samples.

\subsection{ALMA Stacking Analysis}

With CO nondetections constituting one-half the observed sample, it is worth considering whether these sources are detected on average through a stacking analysis. This would imply that somewhat deeper integrations would have been necessary to detect the objects individually. Similarly, although we did not detect (nor expect to detect) dust continuum emission in any individual target, we expect that it should be detectable in a stacked continuum image, if the assumptions made about $\alpha_{\mathrm{CO}}$ in the previous section are correct.

\subsection{1. $2 \mathrm{~mm}$ Continuum Stack}

Because all targets were observed in similar array configurations with similar synthesized beam sizes, for similar durations, and at similar sky frequencies, we opt to simply average together inverted images of the targets rather than perform this analysis in the Fourier plane. The similarity in observed frequency is of particular importance for the continuum stacked image, minimizing the effects of the steep thermal dust spectral index. We create a stacked $2 \mathrm{~mm}$ continuum image simply by averaging together the signal-free continuum maps of each source. In the stacked image, a faint source with $S_{2 \mathrm{~mm}}=17 \pm 5 \mu \mathrm{JY}$ is detected at the center of the field. By subdividing the stack into sources either detected or undetected in $\mathrm{CO}(2-1)$, it is apparent that the signal seen in the full stack is due entirely to the sources individually detected in CO. The CO-detected sources are detected in the $2 \mathrm{~mm}$ continuum stack with $S_{2 \mathrm{~mm}}=23 \pm 7 \mu \mathrm{JY}$, while no continuum emission is detected in the stack of $\mathrm{CO}$ nondetections, with a $3 \sigma$ upper limit $S_{2 \mathrm{~mm}}<25 \mu \mathrm{JY}$.

We can use the detection of $2 \mathrm{~mm}$ continuum emission in the stack of CO-detected sources to cross-check our assumptions about the $\mathrm{CO}-\mathrm{H}_{2}$ conversion factor described in Section 2.3. The average molecular gas mass of the detected objects is $1 \times 10^{10} M_{\odot}$. Assuming a typical dust emissivity, $\kappa_{235 \mathrm{GHz}}=$ $0.42 \mathrm{~cm}^{2} \mathrm{~g}^{-1}$ (e.g., Dunne et al. 2000), where $235 \mathrm{GHz}$ is the rest-frame continuum frequency, a dust temperature $T_{\text {dust }}=$ $25 \mathrm{~K}$, and a gas-to-dust ratio typical of solar-metallicity systems, $\delta_{\mathrm{GDR}}=100$ (e.g., Sandstrom et al. 2013), we find an average molecular gas mass for the $\mathrm{CO}$-detected sources of $8.8 \times 10^{9} M_{\odot}$. This value is in excellent agreement with our CO-based estimates. The same assumptions applied to the $2 \mathrm{~mm}$ upper limit from the $\mathrm{CO}$-undetected sources yield a $3 \sigma$ upper limit of $M_{\mathrm{H}_{2}} 9.6 \times 10^{9} M_{\odot}$, unsurprisingly in agreement with the CO-based upper limits for individual sources. We note that the dust-based estimate of $M_{\mathrm{H}_{2}}$ is also uncertain by about a factor of 2 due to uncertainties in the dust emissivity and massweighted temperature (e.g., Draine \& Li 2007). This independent check indicates that our assumptions about $\alpha_{\mathrm{CO}}$ are reasonable.

\subsubsection{CO Spectral Stack}

We create a stacked $\mathrm{CO}$ image cube in a fashion similar to the simpler stacked continuum maps. In this case, however, we rely on the fact that all targets (detected and undetected) have precisely known redshifts from the LEGA-C spectra. The redshifts are accurate to $\sim 10 \mathrm{~km} \mathrm{~s}^{-1}$, much less than the typical line widths seen in $\mathrm{CO}$ or the stellar absorption features in the LEGA-C spectra. We also do not see significant velocity offsets between the $\mathrm{CO}$ emission and stellar absorption features in the CO-detected sources. We stack the spectra of individual sources using image cubes with velocity resolution ranging from 50 to $800 \mathrm{~km} \mathrm{~s}^{-1}$; the choice of channel width does not affect our conclusions. We have also verified that this procedure accurately recovers the sample-average line flux by stacking only the sources individually detected in CO.

No $\mathrm{CO}(2-1)$ emission is detected in the stack of individually undetected sources, regardless of the velocity resolution used in the stack. As with the individually undetected sources, we place an upper limit on the $\mathrm{CO}$ emission in this stack using a single $800 \mathrm{~km} \mathrm{~s}^{-1}$ wide channel. This results in a $3 \sigma$ upper limit on the CO luminosity $L_{\mathrm{CO}(2-1)}^{\prime}<5.8 \times 10^{8} \mathrm{~K} \mathrm{~km} \mathrm{~s}^{-1} \mathrm{pc}^{2}$. Under the same assumptions about the $\mathrm{CO}$ excitation and $\alpha_{\mathrm{CO}}$ as before, this is equivalent to $M_{\mathrm{H}_{2}}<3.2 \times 10^{9} M_{\odot}$. Given the average stellar mass of the $\mathrm{CO}$-undetected sources, 


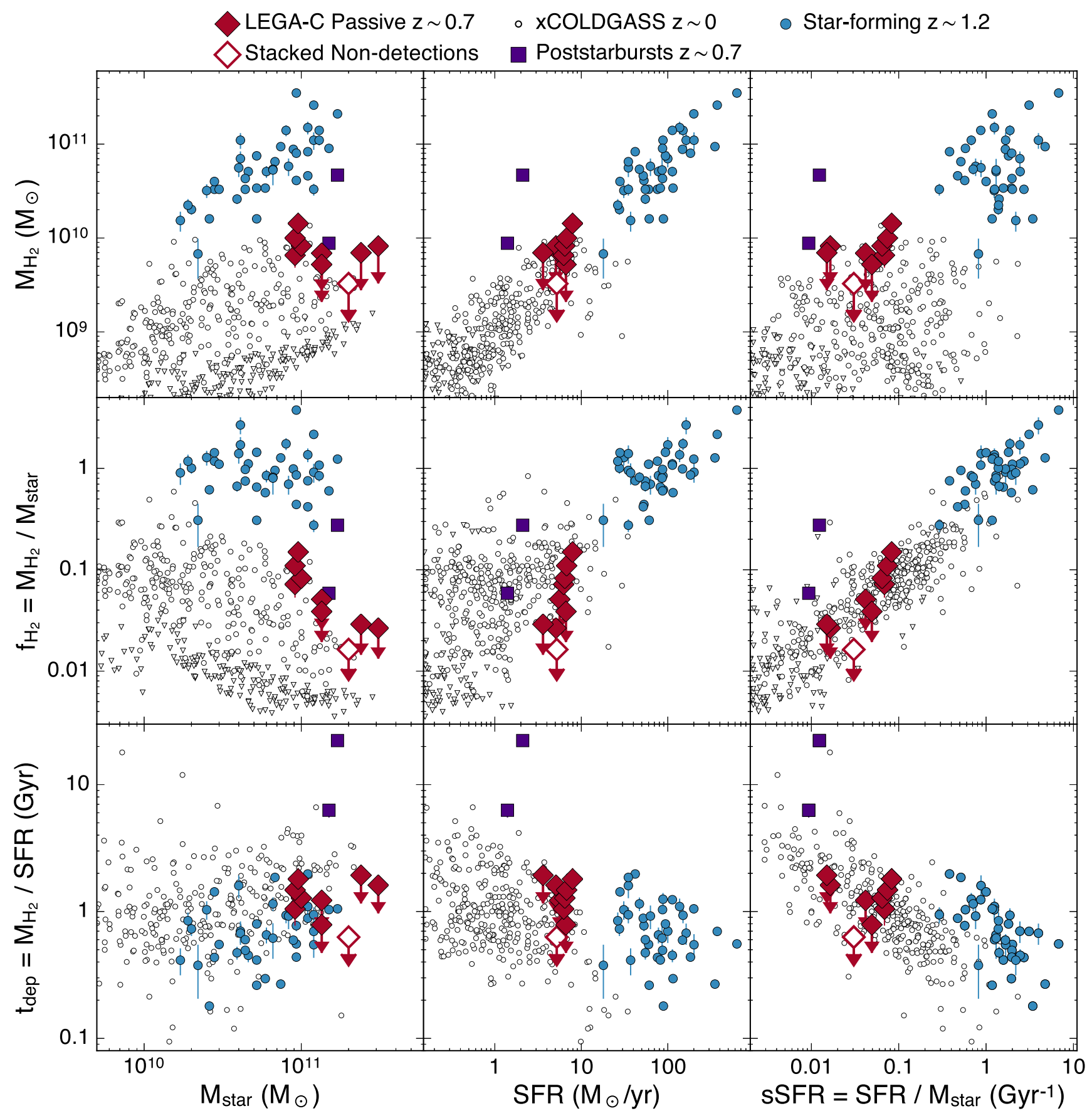

Figure 5. Summary of basic results derived from our data, with comparison samples detailed in the text. From top to bottom, each row plots the molecular gas mass $M_{\mathrm{H}_{2}}$, gas fraction $f_{\mathrm{H}_{2}}$, and gas depletion time $t_{\mathrm{dep}}$; from left to right each column shows the stellar mass $M_{\text {star }}$, star formation rate SFR, and specific SFR. The comparison samples are drawn from Tacconi et al. (2013), Papovich et al. (2016), Saintonge et al. (2017), and Suess et al. (2017). All upper limits are $3 \sigma$. For clarity of presentation, we do not show upper limits for the xCOLDGASS sample in the bottom $\left(t_{\mathrm{dep}}\right)$ row.

$2 \times 10^{11} M_{\odot}$, the resulting upper limit on the gas fraction of these passive sources is $f_{\mathrm{H}_{2}}=M_{\mathrm{H}_{2}} / M_{\text {star }}<0.016$.

\section{Results}

\subsection{Basic Gas Properties}

The derived properties of our sample of $z \sim 0.7$ passive galaxies are given in Table 1. Figure 5 plots a number of basic correlations from our data, comparing galaxy molecular masses, gas fractions, and depletion times with their stellar masses, SFRs, and specific SFRs. We draw comparison samples from the literature. We include the xCOLDGASS sample (Saintonge et al. 2017), a large sample of galaxies in the local universe that contains both star-forming and passive objects over several orders of magnitude in stellar mass. We also include higher-redshift star-forming galaxies at $z=1-1.3$ compiled from the PHIBSS survey (Tacconi et al. 2013), consisting of $\log M_{\text {star }} / M_{\odot}>10.4$ galaxies, supplemented by the observations of Papovich et al. (2016), which extend to lower mass $\left(\log M_{\text {star }} / M_{\odot} \sim 10.2\right)$. Finally, we include the recent ALMA observations by Suess et al. (2017) of two $z \sim 0.7$ galaxies selected to have poststarburst-like optical 
spectra, $\log M_{\text {star }} / M_{\odot} \approx 11$, and little ongoing star formation. With stellar masses similar to our own sample, these galaxies represent objects that quenched recently, as opposed to the several-gigayear-old stellar populations in our sample. Gas masses from all samples have been renormalized to $\alpha_{\mathrm{CO}}=4.4$, but we preserve the original authors' assumptions about the $\mathrm{CO}$ excitation, applicable to the $z>0$ samples.

A number of well-known strong correlations are immediately apparent in Figure 5, including the spatially integrated Schmidt-Kennicutt star formation relation between SFR and $M_{\mathrm{H}_{2}}$ (Schmidt 1959; Kennicutt 1998) and the declining depletion times observed for galaxies with high sSFR (e.g., Saintonge et al. 2017). Because all of these quantities evolve with redshift, it is generally more instructive to interpret the variations seen in Figure 5 after removing or otherwise accounting for the redshift evolution. We perform this exercise in Sections 4.1 and 4.2.

At the most basic level, we observe that $z \sim 0.7$ massive, passive galaxies contain $\lesssim 10^{10} M_{\odot}$ of molecular gas and may contain significantly less, depending on the true masses of the undetected sources. This is nevertheless over an order of magnitude higher $M_{\mathrm{H}_{2}}$ (also $f_{\mathrm{H}_{2}}$ ) than observed for early-type and other massive, low-sSFR galaxies in the local universe (e.g., Combes et al. 2007; Young et al. 2011; Davis et al. 2016). Unlike the local samples, however, we see no evidence for increased gas depletion times that might indicate that the molecular material has been stabilized against collapse; instead, the $\lesssim 1-2$ Gyr depletion times are typical of, or even shorter than, galaxies near the star-forming sequence.

In terms of basic molecular gas properties, the galaxies we have observed are not so dissimilar from "normal" star-forming galaxies in the local universe. While they were selected to be massive galaxies, the absolute values of $M_{\mathrm{H}_{2}}, f_{\mathrm{H}_{2}}$, and $t_{\mathrm{dep}}$ in the "passive" $z \sim 0.7$ galaxies are very similar to star-forming galaxies today. In some sense, the galaxies we have observed can be considered ahead of their time: they formed the bulk of their stellar mass at early times and reached an evolutionary stage similar to that currently experienced by galaxies in the local universe $\sim 6$ Gyr early.

It is rather curious that the detected sources are very clearly detected, at the $\sim 8 \sigma$ level, while the others are not detected even in a stacked spectrum. Why were some objects in our sample detected, and others not? With only eight target galaxies in total, we cannot draw strong conclusions on this point. The four nondetections are both the highest- $M_{\text {star }}$ and lowest-sSFR galaxies in the sample, although the absolute SFRs are not significantly different from the full sample. The nondetections tend to lie toward higher stellar surface density than the detections (Figure 2), but are not obviously disparate in morphology or other structural parameters (e.g., Sérsic index). They also have the lowest $\mathrm{H} \delta$ equivalent widths in the sample and the reddest $U-V$ colors (Figure 3), but do not show large differences in best-fit stellar age, presence or lack of radio $\mathrm{AGNs}$, or presence or lack of emission lines in the LEGA-C spectra. Finally, the undetected sources are in regions more overdense than the detected sources by $0.25 \pm 0.4$ dex. There is no statistical difference in the relative overdensity between detected and undetected sources. We stress that a much larger sample is required to begin to understand which of these properties, if any, are good or useful predictors of CO line luminosity.
We see an interesting contrast between our massive, passive galaxies and the poststarburst galaxies at similar redshift and $M_{\text {star }}$ observed by Suess et al. (2017). While SFRs for the poststarburst sample are measured from the extinctionsusceptible [O II] doublet, and thus may be underestimated, the absolute quantity of molecular gas in these two objects still serves as a useful reference. While one poststarburst has a molecular mass similar to the objects we have studied, the other has $>2 \times$ higher $M_{\mathrm{H}_{2}}$ than any of our objects, and more than an order of magnitude higher $M_{\mathrm{H}_{2}}$ than the stack of CO-undetected sources in our sample. If the SFR of this object is confirmed to be as low as inferred, it would indicate a large diversity in the gas masses, fractions, and depletion times among massive galaxies at intermediate redshifts.

\subsection{Molecular and Stellar Dynamics}

The dynamical state of the molecular gas in passive galaxies can be a powerful probe of its origin and the processes that led to the cessation of star formation. In $z \sim 0$ early-type galaxies, for example, approximately one in four contain detectable amounts of molecular gas, much more common among the fast rotator galaxies than the slow rotator population (Young et al. 2011). Among these objects, about $40 \%$ show signs that the molecular gas disks are significantly misaligned (more than $30^{\circ}$ ) with respect to the stellar rotation (Alatalo et al. 2013; Davis et al. 2013). This has been interpreted as evidence that the molecular gas was gained from external sources after the galaxies quenched star formation, for example from the accretion of gas-rich satellites or cold streams from the intergalactic medium. Misaligned rotation axes can also arise during major mergers if gas makes up a significant fraction of the total mass (Lagos et al. 2018). The dynamics of the molecular gas can thus provide insight into the origin of this material and its relationship to the physical processes that quenched star formation.

From the integrated spectra alone, two of our CO-detected objects show double-horned line profiles characteristic of rotating gas disks, while the other two show centrally peaked line profiles. This is similar to the results from local early-type galaxies, for which $>30 \%$ show evidence of rotation from the CO line profiles alone (Young et al. 2011). The remaining objects may also contain rotating gas disks, depending on the spatial distribution of the molecular gas and the inclination with respect to the line of sight.

To investigate the velocity fields of the CO-detected objects in more detail, we reimaged the ALMA data, splitting the total $\mathrm{CO}$ emission from each object into two velocity channels covering the red- and blueshifted emission. The results are shown in Figure 6 (top row). While narrower velocity bins can also be imaged, a single blue and red channel yields the highest signal-to-noise ratio for presentation purposes; we have verified that the velocity gradients apparent in Figure 6 are consistent with imaging the data in narrower velocity channels. We fit the red and blue channels of each source in both the visibility and image domains with point-source models, finding that the centroids of each velocity component can be determined to $\approx 0$." 3 , on average. Given the uncertainties, significant velocity gradients are apparent in three of the four detected sources (IDs 74512, 110509, and 130284). The remaining source (ID 132776) may also show a velocity gradient in $\mathrm{CO}$, but the spatial separation between blueshifted and redshifted velocities is not significant at the signal-to-noise ratio of the current data. 

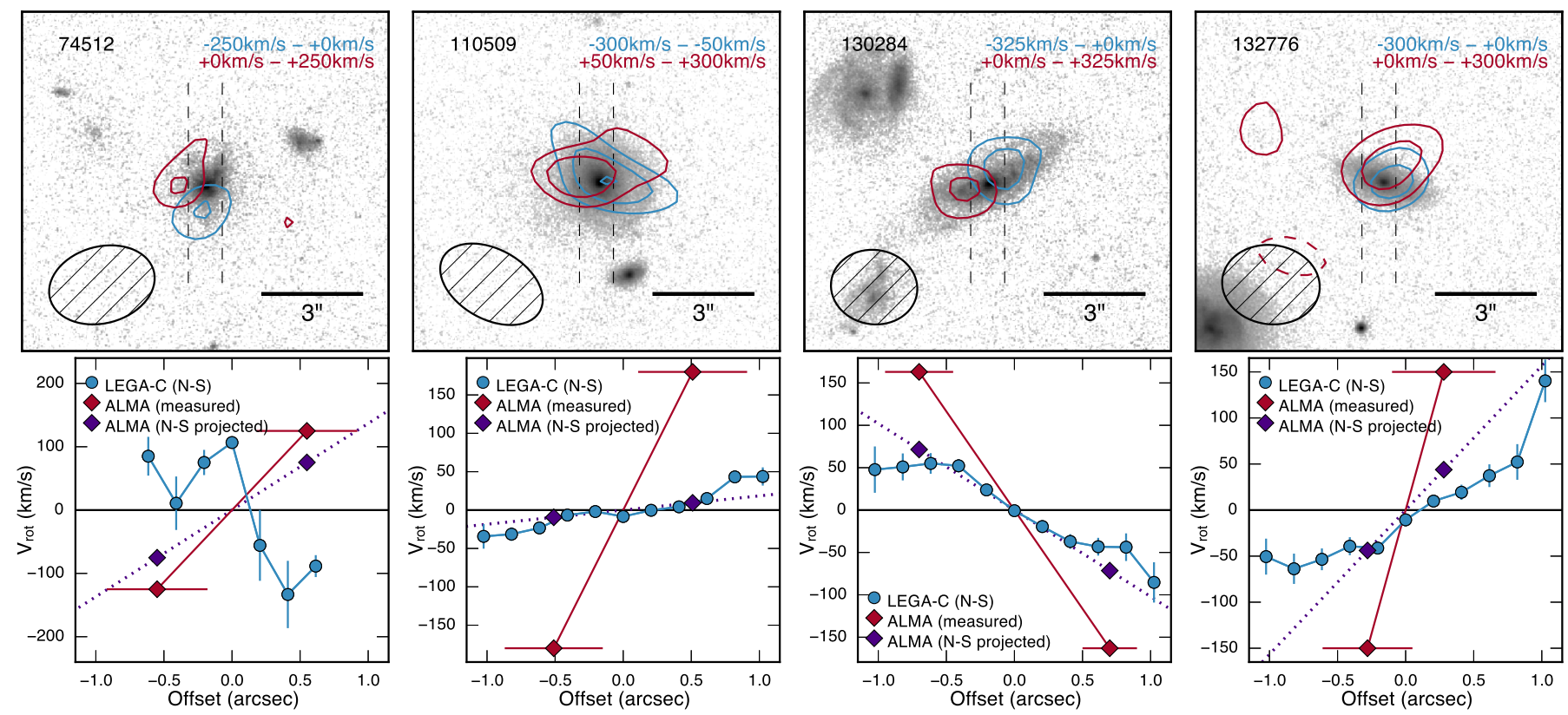

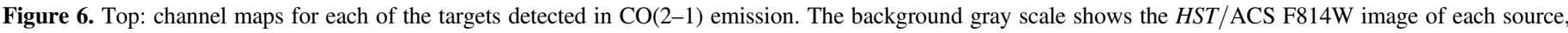

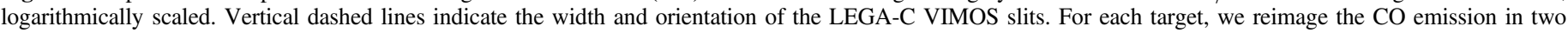

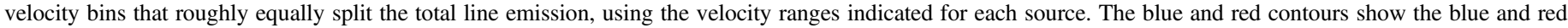

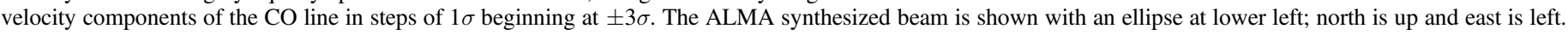

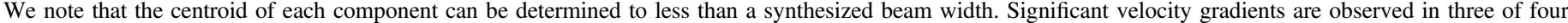

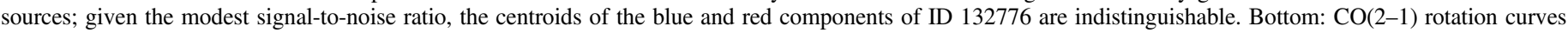

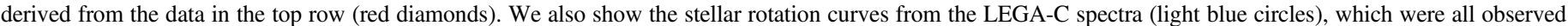

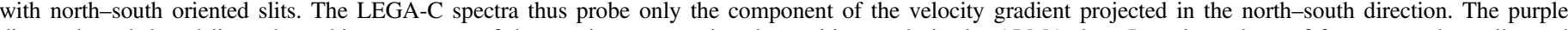

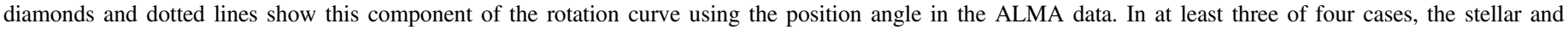
molecular rotation axes are not obviously misaligned.

Our finding that a high fraction of $z \sim 0.7$ passive galaxies show measurable rotation mirrors the results for higher-SFR massive galaxies at equivalent and higher redshifts (e.g., Förster Schreiber et al. 2009; Tacconi et al. 2013; Wuyts et al. 2016).

A quantitative comparison between the molecular gas and stellar dynamics is challenging given the data in hand. The LEGA-C spectra provide a high signal-to-noise measurement of the stellar rotation curve (Bezanson et al. 2018), but all VIMOS slits for the ALMA sample were $1^{\prime \prime}$ wide and oriented north-south, and so are effectively randomly aligned with respect to the galaxy major axes. Additionally, the typical $\sim 1^{\prime \prime}$ seeing during the VLT observations leads the rotation curve velocities to be correlated on scales of a kiloparsec. On the other hand, the ALMA CO observations have both lower spatial resolution $\left(\sim 2^{\prime \prime}-2{ }^{\prime \prime} 5\right)$ and lower signal-to-noise ratio than the LEGA-C spectra, but contain full two-dimensional spatial information and independent velocity channelization.

Given the differences between the available data and the modest signal-to-noise ratio of the current $\mathrm{CO}$ maps, we choose not to make a detailed comparison between the $\mathrm{CO}$ and stellar dynamics. Instead, we address a simpler question: are the LEGA-C stellar rotation curves consistent with the observed $\mathrm{CO}$ velocity gradients, given the misalignment between the projected galaxy rotation axes and the north-south oriented VIMOS slits? In Figure 6 (bottom row), we show the velocity gradients seen in the $\mathrm{CO}$ data, where the offset between red and blue halves of the emission is measured along the galaxy rotation axis derived from the ALMA data. We also show the LEGA-C stellar rotation curves, which, as mentioned, are sensitive only to the north-south projected component of the velocity field. Finally, we show the portion of the ALMA velocity gradient also projected onto the north-south orientation of the LEGA-C slits.

In three of four cases, we find excellent agreement between the LEGA-C rotation curve and the north-south projection of the $\mathrm{CO}$ velocity gradient. The final object, ID 74512 , is the most compact galaxy in the sample and does not obviously show signs of rotation in the LEGA-C spectrum. This may be at least in part due to the compact size of the galaxy in comparison to the VIMOS slit and the low signal-to-noise ratio of the stellar rotation curve. It is also possible that the stellar component of this galaxy truly has little rotation, which would imply that the angular momentum axes of the stars and molecular gas are misaligned. Given the large uncertainties involved, however, this scenario is neither supported nor unsupported by the data in hand.

In summary, the rotational axes of the stellar and molecular components of the CO-detected objects are consistent in at least three of four cases. This argues against an external origin for the molecular gas in $z \sim 0.7$ passive galaxies (either recently accreted cold streams or gas-rich mergers), because these processes should commonly result in misaligned stellar and molecular rotation. Instead, the molecular gas in our sample is probably left over from the formation epoch of the bulk of the galaxies' stellar mass or replenished directly by stellar mass loss, resulting in stellar and gas disks with aligned rotational axes. 


\section{Discussion}

\subsection{Gas Scaling Relations}

The past decade has seen a large investment of single-dish and interferometer time devoted to understanding how the molecular gas properties of galaxies vary with other galaxy properties from $z \sim 0$ (e.g., Bothwell et al. 2014; Saintonge et al. 2017) out to $z \sim 2.5$ (e.g., Tacconi et al. 2013; Genzel et al. 2015; Scoville et al. 2017; Tacconi et al. 2018). Because of the well-known correlation between $M_{\mathrm{H}_{2}}$ and SFR (Figure 5), the moderate- to high-redshift samples have focused nearly exclusively on fairly massive star-forming galaxies on or above the star-forming sequence, the objects with the largest gas masses and most easily detectable. With our sample of passive galaxies at intermediate redshifts, we are in a position to determine whether these scaling relations extend to lower sSFR, a previously unexplored parameter space.

The above studies provide prescriptions for the variation of molecular gas fraction $f_{\mathrm{H}}$ and depletion time $t_{\mathrm{dep}}$, parameterized in terms of overall redshift evolution, sSFR (generally with respect to the sSFR expected from the star-forming sequence at a given epoch), $M_{\text {star }}$, or galaxy size $r_{\text {eff. }}$. Based on samples of hundreds of galaxies in the range $z=0-3$, consensus has emerged that the gas fraction $f_{\text {gas }}$ evolves steeply with redshift, $\propto(1+z)^{1.8-2.5}$, with shallower dependencies on starforming sequence offset, $\propto(\Delta \mathrm{SSFR})^{0.3-0.5}$, and stellar mass, $\propto\left(M_{\text {star }}\right)^{-(0.3-0.7)}$. The evolution of $f_{\text {gas }}$ with redshift appears to be slightly less rapid than the normalization of the star-forming sequence itself. This implies that to first order, the higher SFRs observed in typical galaxies at high redshift are simply due to larger gas masses, with perhaps a somewhat higher efficiency of star formation (equivalently, lower $t_{\mathrm{dep}}$ ) also needed. The depletion time $t_{\text {dep }}$ exhibits an overall smaller dynamic range and varies less steeply with redshift, $\propto(1+z)^{-(0.3-1)}$, and star-forming sequence offset, $\propto(\Delta \mathrm{sSFR})^{-(0.4-0.7)}$, and has very shallow dependence on stellar mass, $\propto\left(M_{\text {star }}\right)^{0-0.17}$. Because $t_{\mathrm{dep}} \approx 1 \mathrm{Gyr}$ for star-forming galaxies, with shallow redshift evolution, galaxies must also have had high gas accretion rates in order to reconcile $t_{\mathrm{dep}}$ with the evolution of $f_{\mathrm{H}_{2}}$, reaching $>100 M_{\odot} \mathrm{yr}^{-1}$ at $z>2.5$ (Scoville et al. 2017).

It is not clear whether these scaling relations should or do extend to passive galaxies significantly below the star-forming sequence. On one hand, the scaling relations are very successful over a very wide parameter space, with residual scatter in $f_{\mathrm{H}_{2}}$ and $t_{\mathrm{dep}}$ of just $\sim 0.1$ dex (Tacconi et al. 2018). On the other hand, the scaling relations have been derived using only star-forming galaxies and thus may not account for the physical mechanisms that quench galaxies or the diversity of these mechanisms that may induce increased scatter in the relations.

In Figure 7, we compare the gas fractions and depletion times for our observed sample with two empirical scaling relations from the literature. ${ }^{17}$ The relations derived by Scoville et al. (2017) are based on ALMA observations of longwavelength dust continuum emission and assumptions about the dust emissivity. The work by Tacconi et al. (2018), on the other hand, is a meta-analysis of studies that represents the

\footnotetext{
17 Both Scoville et al. (2017) and Tacconi et al. (2018) provide scaling relations using the prescription for the star-forming sequence of Speagle et al. (2014), which we also adopt for Figure 7. The difference between the Speagle et al. and Whitaker et al. (2012) formulations explains why two objects in our sample are $>10 \times$ below the star-forming sequence, lower than our nominal selection threshold.
}

largest overall sample size and dynamic range to date, and also accounts for different normalizations in the major techniques used to measure gas masses. Tacconi et al. (2018) adopt a metallicity-dependent $\mathrm{CO}-\mathrm{H}_{2}$ conversion factor based on the observed mass-metallicity relation, while we simply use $\alpha_{\mathrm{CO}}=4.4$. We note that using their prescription for our sample gives a smaller value for $\alpha_{\mathrm{CO}}$, resulting in even lower gas masses and fractions and shorter depletion times; in other words, the differences seen in Figure 7 would be even further accentuated.

The darkly shaded regions in Figure 7 show the approximate lowest sSFR objects at $z \sim 0.7$ contained in the observational samples; at lower sSFR (lightly shaded), the scaling relations are extrapolated. For both $f_{\mathrm{H}_{2}}$ and $t_{\text {dep }}$, Figure 7 shows that the extrapolation of the Tacconi et al. (2018) scaling relation (which also incorporates the data from Scoville et al. 2017) is a better match to our data, while the Scoville et al. (2017) relations predict significantly higher $f_{\text {gas }}$ and longer $t_{\text {dep }}$ compared to our observations. The Tacconi et al. (2018) scaling relations perform reasonably well for the CO-detected sources closest to the star-forming sequence. However, both scaling relations perform poorly for the $\mathrm{CO}$-undetected sources in our sample, especially so for the stacked nondetections. Individual nondetections have upper limits on $f_{\text {gas }}$ lower than the extrapolations by $\sim 2 \times$, while the upper limit on $f_{\text {gas }}$ in the stack is a factor of 5 lower than expected. A similar discrepancy applies to $t_{\mathrm{dep}}$, with individual sources showing depletion times shorter by $1.6-2.5 \times$ and the stack too short by a factor of 4 .

Considering either $f_{\mathrm{H}_{2}}$ or $t_{\mathrm{dep}}$, the CO-undetected sources contain significantly less molecular gas than expected. This result holds even if we have overestimated the SFRs in our sample by $\gtrsim 5 \times$, because the scaling of $f_{\mathrm{H}_{2}}$ with sSFR is very steep, and altering the SFR moves galaxies largely parallel to the $t_{\text {dep }}$ scaling relation. At the sSFRs of our sample, we expect the SFRs to be overestimated by at most about a factor of two (Fumagalli et al. 2014, and Section 2.1). It is interesting to note that the four undetected sources are the four farthest below the star-forming sequence, but with a small sample size it is unclear that this is significant (Section 3.1).

It is apparent that the literature scaling relations cannot be extrapolated and lose predictive power at the low sSFRs probed by our sample. Instead, there appears to be either a break in the scaling relations toward lower $M_{\mathrm{H}_{2}}$ at sSFRs $4-5 \times$ below the star-forming sequence, or a sharp increase in the scatter of the scaling relations, with our small sample size explaining the lack of detections closer to the expected relations. Equivalently, a curvature term in the $\Delta$ (sSFR) dependency could be required in the scaling relations in order to bring them into agreement with our observations. Only a larger sample of galaxies well below the star-forming sequence would reveal whether this is necessary.

It is not clear why half of the observed sample deviates so significantly from the extrapolated scaling relations. If this trend is real, the answer likely involves the physics of galaxy quenching, including feedback processes and galactic winds, gas starvation or lowered accretion rates, environmental effects, or morphological transformation. One or more of these processes likely acted on the observed galaxies in order to disrupt their equilibrium growth, leaving them with significantly less molecular gas than otherwise expected. We return to this question in Section 5.1. 


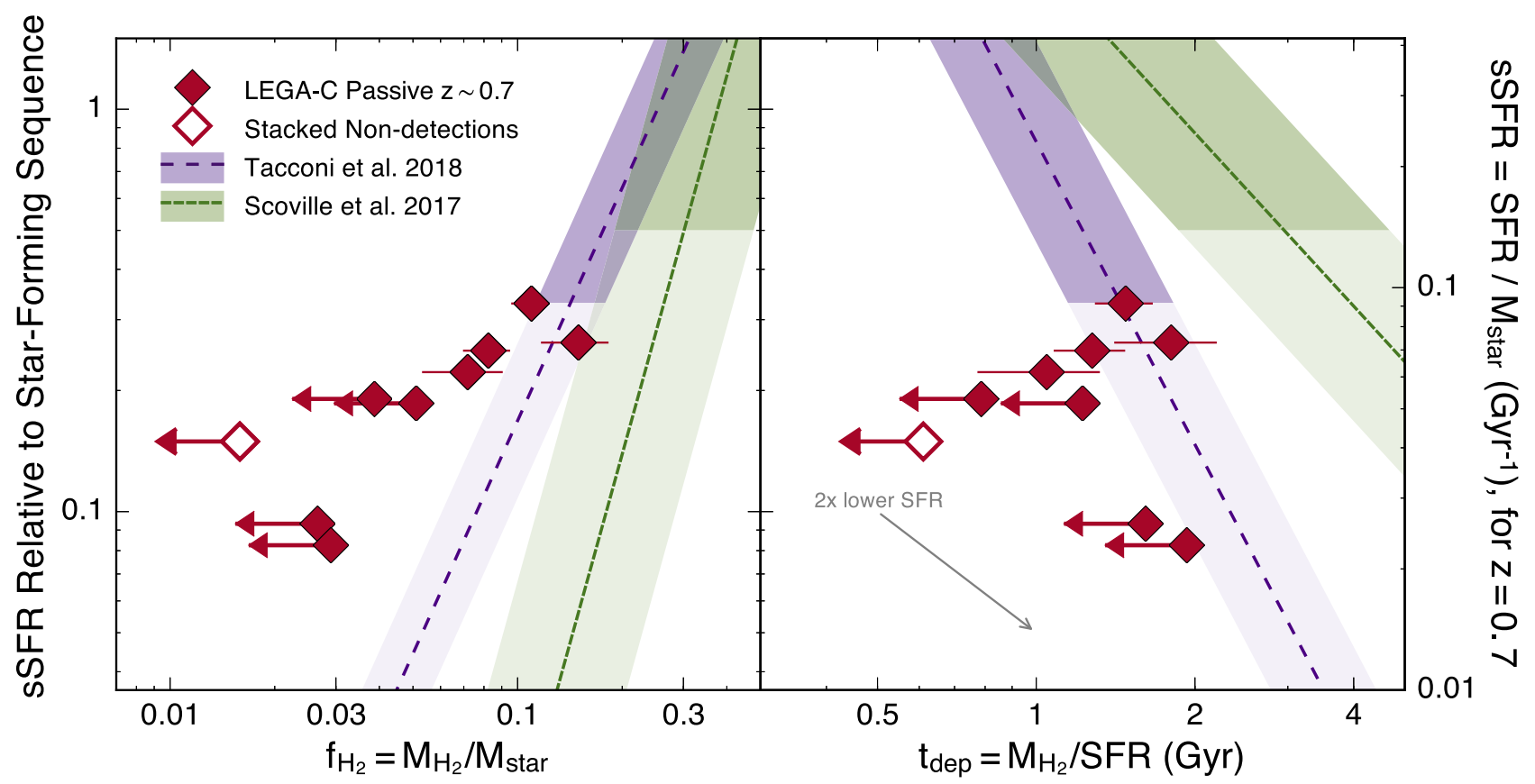

Figure 7. Molecular gas fraction $f_{\mathrm{H}_{2}}$ (left) and depletion time $t_{\text {dep }}$ (right) vs. the specific SFR, compared with observationally derived literature scaling relations from Scoville et al. (2017; green densely dashed line and region) and Tacconi et al. (2018; purple dashed line and region), evaluated for $\log M_{\text {star }} / M_{\odot}=11$. Both literature scaling relations are parameterized in terms of the offset of the sSFR with respect to the expectation from the star-forming sequence, so the left-hand $y$ axis is also labeled in these terms. The right-hand $y$ axis is labeled with the corresponding values of the sSFR, evaluated at $z=0.7$. The shaded regions encompass the quoted approximate $1 \sigma$ residual scatter around the relations. The darker shaded bands correspond to the approximate sSFR lower bounds for galaxies at $z=0.6-0.8$ that were used in the derivation of the scaling relations; below these regions the scaling relations are extrapolations (lightly shaded). In the right-hand panel, a gray arrow indicates the shift if the SFRs for our galaxies have been overestimated by a factor of 2; the shift is largely parallel to the scaling relations. In general, the CO-detected objects in our sample are in reasonable agreement with the scaling relations, while the undetected objects, and especially the stacked nondetections, are significantly offset from the extrapolated relations. This could indicate either a strong break in the scaling relations at $3-5 \times$ lower sSFR than the star-forming sequence, or a large increase in the scatter of $f_{\mathrm{H}_{2}}$ and $t_{\mathrm{dep}}$.

\subsection{Comparison to Cosmological Simulations}

Recent large cosmological simulations have been able to produce populations of galaxies with properties broadly consistent with many observations (e.g., Vogelsberger et al. 2014; Schaye et al. 2015). Typical target benchmarks include matching the galaxy stellar mass function at $z=0$ and higher redshifts, the star formation history of the universe, galaxy colors, and other observables. The generally good agreement between the simulations and these basic observed properties then allows us to explore more detailed aspects of the simulations, which can help probe whether the subgrid physical processes modeled by the simulations are accurately calibrated. Passive galaxies in particular are useful in this comparison, as they are the end products of powerful galactic feedback and quenching processes that encapsulate the most significant differences between various simulations. Here we compare the molecular gas properties of our observed $z \sim 0.7$ passive galaxies to predictions from the Evolution and Assembly of GaLaxies and their Environments (EAGLE) simulation (Crain et al. 2015; McAlpine et al. 2016; Schaye et al. 2015).

Molecular gas masses for galaxies in the Ref-L100N1504 EAGLE run were calculated by Lagos et al. $(2015,2016)$ using two different prescriptions for the molecular fraction that depend on, for example, the metallicity, density, pressure, and SFR of each gas particle (Gnedin \& Kravtsov 2011; Krumholz 2013). In comparing the simulated galaxies' SFRs and gas masses with observations at $z=0$, Lagos et al. (2015) find good agreement for "normal" star-forming galaxies. On the other hand, EAGLE underpredicts the number of gas-rich early-type galaxies compared to observations; early-type galaxies from the ATLAS ${ }^{3 \mathrm{D}}$ survey are overrepresented at the gas-rich end compared to the simulation, with $17 \%$ of observed galaxies lying $>2 \sigma$ from the median $\mathrm{SFR}-M_{\mathrm{H}_{2}}$ relation in EAGLE. Although no morphological selection of early types was performed in the simulation, clouding the comparison, the difference could indicate that the star formation "law" employed by EAGLE is too simplistic.

In Figure 8 we make a similar comparison to the simulations using our ALMA observations. This figure shows the SFR $-M_{\mathrm{H}_{2}}$ star formation relation using a $z=0.6$ snapshot from EAGLE; we obtain a virtually identical result using a $z=0.85$ snapshot. For this comparison, we simply average the results from the two prescriptions used by Lagos et al. (2015) to calculate $M_{\mathrm{H}_{2}}$ for the simulated galaxies; using one or the other alone also has no impact on our conclusions. The blue line and shaded region in Figure 8 show the median and $90 \%$ interval of SFR as a function of $M_{\mathrm{H}_{2}}$ for all galaxies in EAGLE with $\log M_{\text {star }} / M_{\odot}>10$, approximately the stellar mass cutoff of the LEGA-C survey.

Making a clean selection of simulated galaxies similar to our own selection criteria for ALMA observations is difficult. While we primarily selected objects from the LEGA-C survey to have sSFRs $3-10 \times$ below the star-forming sequence at $z \sim 0.7$, it is known that EAGLE does not accurately track the evolution of the star-forming sequence to high redshifts. In particular, the simulated galaxies underpredict the normalization of the star-forming sequence by $\sim 0.2-0.4$ dex at $z=1$ (Furlong et al. 2015). This issue is not unique to EAGLE; the 

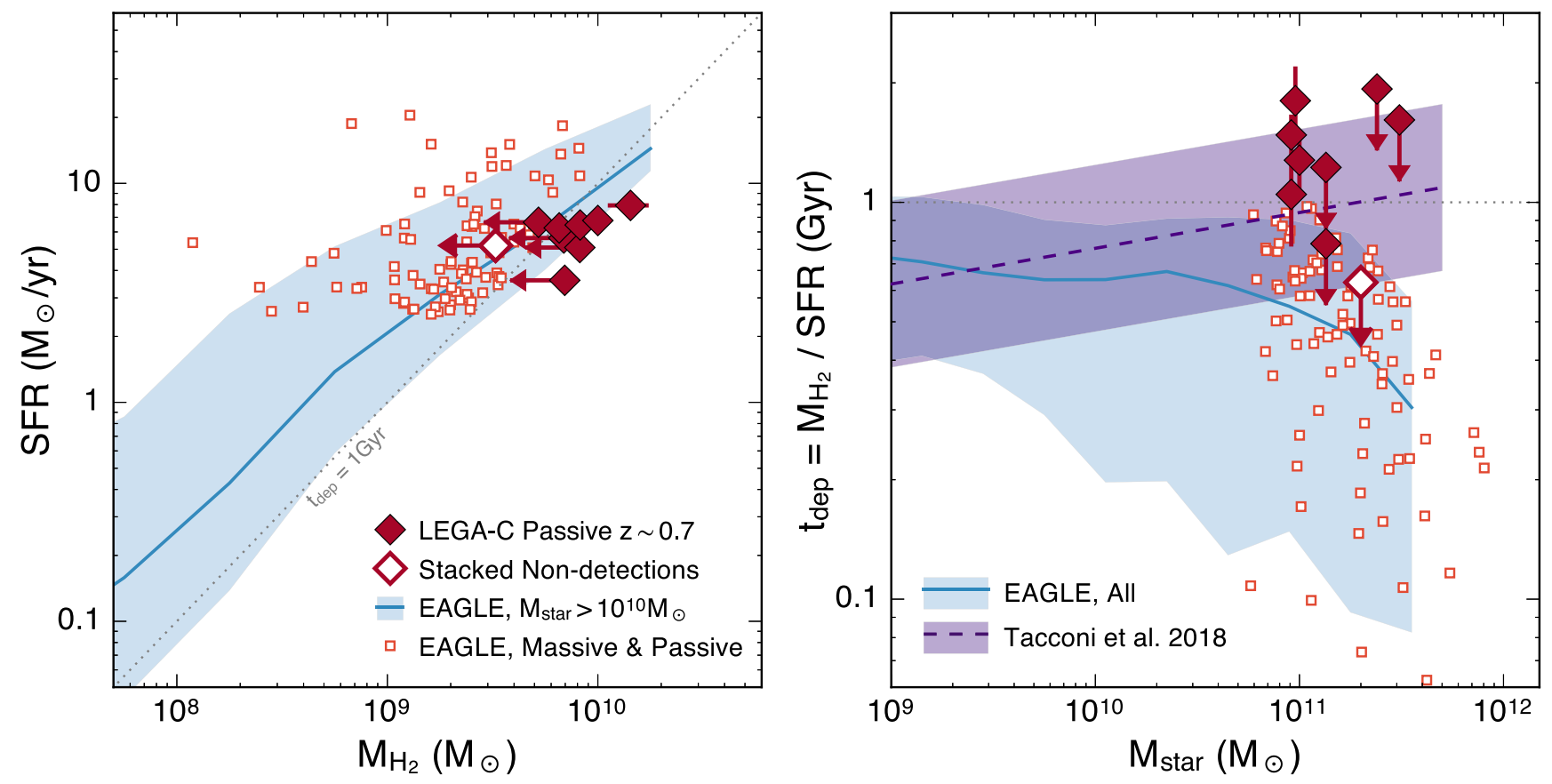

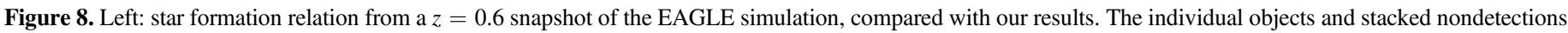

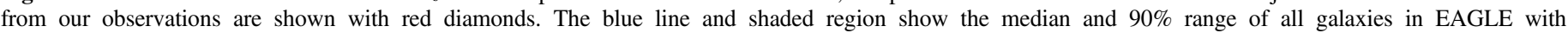

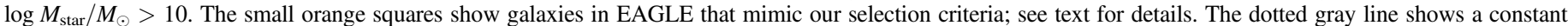

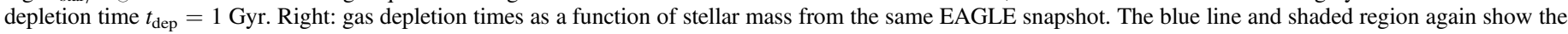

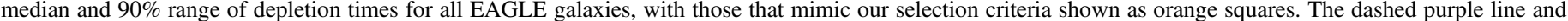

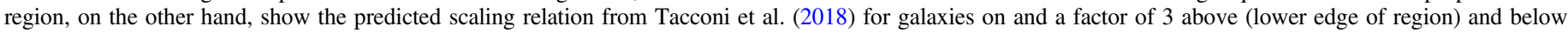

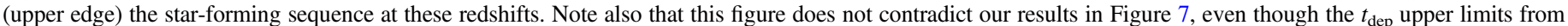
the $\mathrm{CO}$ nondetections overlap with the shaded region, because these galaxies have significantly lower sSFR than merely $3 \times$ below the star-forming sequence.

same is seen in other cosmological simulations (e.g., Illustris; Sparre et al. 2015). It is also worth noting that even the observations are not self-consistent on this point, with the integral of the cosmic SFR density overpredicting the observed stellar mass density by $\sim 0.2$ dex (e.g., Madau \& Dickinson 2014, though see also Driver et al. 2018), while the simulations obviously must be self-consistent. Given the uncertainty in the normalizations of these relations in the literature, instead of selecting galaxies with the same range of sSFRs as the observations, we instead simply select the objects with sSFRs lower than $95 \%$ of the total population of simulated galaxies. Although the quantitative selection is different, this preserves the spirit of our selection, aiming for objects much more passive than the overall population. We also restrict the comparison to galaxies with $M_{\mathrm{H}_{2}}>10^{7} M_{\odot}$, below which gas masses are unreliable due to the baryonic particle mass resolution of EAGLE. We preserve our other two main selection criteria, $\log M_{\text {star }} / M_{\odot}>10.8$ and $\mathrm{SFR}>$ $2.5 M_{\odot} \mathrm{yr}^{-1}$, as in the observed sample, resulting in 91 simulated massive, passive galaxies. While these are absolute, rather than relative, selection cuts, they have little influence on our conclusions because, as we will show, massive and passive galaxies in EAGLE do not show a systematically different starformation relation compared to the rest of the simulated galaxies. The objects mimicking our selection are shown with small orange squares in Figure 8.

We find that our observed sample tends to lie near, but slightly offset from, the bulk of simulated galaxies. The COdetected sources, in particular, seem to have either too much molecular gas given their SFRs, or too little star formation given the amount of gas they contain. In other words, their depletion times are slightly longer than seen in the simulation.
We draw the same conclusion from comparing only to those objects that mimic our selection criteria, which are not systematically offset from the rest of the galaxies in the simulation. The differences would be further exaggerated if the SFRs in the simulation or observations have been poorly estimated-either too low in EAGLE due to the underprediction of the star-forming sequence, or too high in our targets due to non-SF dust heating at $24 \mu \mathrm{m}$. If the SFRs of the observed and comparison simulated galaxies are generally correct, however, it appears that the differences are mostly in $M_{\mathrm{H}_{2}}$ and not in SFR. The simulated galaxies have SFRs comparable to the observed sample (partly by construction, due to our SFR selection threshold), but a median $M_{\mathrm{H}_{2}}$ $=2 \times 10^{9} M_{\odot}$, with a dispersion of $0.2 \mathrm{dex}$ and a tail that extends to lower molecular masses. Indeed, even the most gasrich EAGLE galaxy matching our selection criteria has lower $M_{\mathrm{H}_{2}}$ than any of our CO-detected objects.

Amusingly, unlike the observationally derived scaling relations of Section 4.1 , in this comparison it is the COundetected LEGA-C objects that are in better agreement with the scaling relation. While we have only upper limits on the depletion time of these objects, they would be consistent with the EAGLE galaxies even if they contained more than an order of magnitude less molecular gas than our present upper limits.

The better agreement for the nondetections appears to be due to the fact that EAGLE predicts a weak decline of $t_{\text {dep }}$ toward high masses, in contrast to the increasing depletion times for massive galaxies predicted by the Tacconi et al. (2018) scaling relations. In EAGLE, the decline in $t_{\text {dep }}$ is largely due to two linked effects. First, the probability distribution function of ISM gas densities is shifted to higher mean density in more massive galaxies. Second, EAGLE assumes a superlinear 
Schmidt-Kennicutt star formation relation. Combined with the higher gas densities in more massive galaxies, this leads to more star formation per unit molecular gas (and lower depletion times) at high masses. This effect would be muted if instead a linear relationship between SFR and $M_{\mathrm{H}_{2}}$ were used in the simulations. EAGLE therefore does not and cannot reproduce the diversity of $t_{\mathrm{dep}}$ values that are unveiled by our observations of passive galaxies, suggesting that adopting a universal Schmidt-Kennicutt relation in the simulation is not sufficient to capture the complexity these observations reveal.

In Figure 8 (right), we examine the depletion times explicitly, comparing the predicted correlation between $t_{\mathrm{dep}}$ and $M_{\text {star }}$ from Tacconi et al. (2018) with that seen in the EAGLE simulation, where the purple shaded region corresponds to the prediction for galaxies a factor of 3 below (upper edge) and above (lower edge) the star-forming sequence at these redshifts. The two scaling relations predict opposite behavior for highmass galaxies. The differences are especially stark for massive and passive galaxies (again shown with orange squares), which follow the same envelope as star-forming galaxies in EAGLE but are predicted to have depletion times longer by a factor of $>5 \times$ than the Tacconi et al. (2018) scaling relation.

Our observations, in summary, provide some support for both the observationally and theoretically derived scaling relations. While the gas-rich, $\mathrm{CO}$-detected objects in our sample agree reasonably well with the extrapolations from Tacconi et al. (2018), our undetected objects have depletion times that are much more consistent with the EAGLE simulation at these redshifts (Lagos et al. 2015, 2016). This highlights the importance of building a larger sample of passive galaxies with molecular mass estimates, which would indicate whether one or both of these scaling relations should be revised; how these scaling relations should be revised is unclear without additional information from a larger sample.

\section{Implications for Galaxy Quenching}

\subsection{Active and Dynamical Quenching}

In contrast to the nearly linear relationship between $M_{\text {star }}$ and SFR observed for lower-mass galaxies from $z=0$ to $z \approx 2.5$, high-mass galaxies $\left(\log M_{\text {star }} / M_{\odot} \gtrsim 10.5\right)$ tend to show a sublinear relationship (e.g., Whitaker et al. 2014; Schreiber et al. 2015). This is generally interpreted as an effect of the "mass quenching" of high-mass galaxies that results in a nearly constant break in the stellar mass function at $\log M_{\text {star }} / M_{\odot} \sim 10.5-11$ (Peng et al. 2010). The various physical mechanisms that cause this mass quenching predict different observable properties of the ISM in passive galaxies and can potentially be disentangled by observations of large samples of massive, quenched objects such as those presented here.

At $z \lesssim 1$, the local environment of a galaxy is correlated with whether or not it is quenched, with galaxies in dense regions much more likely to be quiescent than those in less dense environments (e.g., Sobral et al. 2011; Darvish et al. 2016). Numerous physical mechanisms can explain this environmental dependence, including ram pressure stripping, starvation, and tidal interactions. The effects of "maintenancemode" AGN feedback are also most prominent in denser environments (Correa et al. 2018). We found no difference in local overdensity between the CO-detected and undetected galaxies, although the large uncertainties in individual estimates of the local overdensities and the small sample size of our study make it difficult to determine whether environmental effects are responsible for the variations in $f_{\text {gas }}$ and $t_{\text {dep }}$ we observe in our sample.

Dynamical processes can suppress the SFR in massive galaxies below the expected rates (e.g., Martig et al. 2009; Genzel et al. 2014), even in the presence of large molecular masses, generally referred to as morphological or gravitational quenching. In this scenario, the formation of a stellar bulge stabilizes an embedded gas disk against collapse, and the stabilization can proceed from the inner regions of a galaxy outward as the stellar surface density increases over time. These dynamical processes can accommodate relatively high gas fractions, up to $f_{\mathrm{H}_{2}} \sim 0.1$, but predict long depletion times reaching $10 \mathrm{Gyr}$ or more; the molecular material is still present within the galaxies but is prevented from forming stars.

Active internal processes, on the other hand, such as feedback from AGNs or star formation, act to suppress star formation by heating or removing the molecular material (see Fabian 2012 for a review). The energetics and driving mechanisms of these feedback processes are being actively debated, but generally predict low cold gas fractions and short depletion times.

Our observations reveal low gas fractions, $f_{\mathrm{H}_{2}} \lesssim 0.1$, and short depletion times, $t_{\text {dep }} \lesssim 2$ Gyr. The CO-undetected objects, in particular, show lower gas fractions and depletion times comparable to or shorter than expected for star-forming galaxies of similar mass, a conclusion that is robust even if the SFRs have been overestimated by $\sim 5 \times$. This demonstrates that intermediate-redshift passive galaxies are genuinely gas-poor, and the low SFRs are not the result of increasing $t_{\text {dep }}$. This suggests that dynamical processes are not acting to suppress the star formation in this sample. A similar conclusion was reached by Sargent et al. (2015) at slightly higher redshift; they failed to detect a single very massive galaxy at $z \sim 1.5$ with $\log M_{\text {star }} / M_{\odot} \sim 11.8$ and concluded that gravitational stabilization of gas disks is not common among the most massive high-redshift, quiescent galaxies. On the other hand, Gobat et al. (2018) concluded that star formation indeed proceeds less efficiently by stacking dust continuum observations of a large number of $z \sim 1.8$ galaxies more quiescent than those we consider here.

Although there is some evidence that the morphological quenching scenario is consistent with the $z=0$ early-type population (Martig et al. 2013), we do not find evidence of it at higher redshift. There are several key differences between our sample and the local massive early types that are likely responsible for this. First, our (detected) sample galaxies contain $\approx 1-2$ orders of magnitude more molecular gas than the $z=0$ population, and the upper limits on the undetected galaxies are still consistent with $>1$ order of magnitude higher $M_{\mathrm{H}_{2}}$. While morphological quenching can accommodate gas fractions of $f_{\mathrm{H}_{2}} \sim 0.1$, this may be difficult for galaxies to achieve in practice without resuming star formation. Second, our galaxies have stellar ages several gigayears younger than the $z=0$ early types (Wu et al. 2018; Chauke et al. 2018) and so are much closer to their epoch of quenching. Martig et al. (2009) show that morphological quenching can act when a galaxy has already assembled a stellar spheroid and nearly depleted its gas reservoir, but is slowly reaccreting gas. It is not clear that the same should hold if the galaxy never fully 
depletes its gas supply, but merely continues to form stars from its existing gas reservoir.

\subsection{Connection to Local Quiescent Galaxies}

Finally, we consider the possible connection between the passive galaxies we have observed at intermediate redshifts and their counterparts in the local universe. Given the molecular masses we have observed, it is immediately clear that if these objects are indeed progenitors of local massive, quiescent galaxies, they must consume, expel, or heat $\gtrsim 90 \%-99 \%$ of the molecular material over the next $6 \mathrm{Gyr}$ in order to match the range of $M_{\mathrm{H}_{2}}$ seen in nearby ellipticals. This implies a much steeper drop in gas fraction compared to star-forming galaxies over the same redshift range, which decline by less than an order of magnitude over the same interval (e.g., Tacconi et al. 2018).

Part of the reason for the slower decline in $f_{\mathrm{H}_{2}}$ for the starforming population is that galaxies continue to accrete substantial amounts of gas over time from minor mergers and streams along the cosmic web (e.g., Kereš et al. 2005). Because $t_{\text {dep }}$ is observed to be much less than a Hubble time, galaxies' gas reservoirs must be replenished if they are to continue forming stars at the observed high rates. The implied gas accretion rates are $\approx 100 M_{\odot} \mathrm{yr}^{-1}$ at $z>2$, declining by a factor of $\sim 7$ from $z=2$ to $z=0.7$, and a further factor of $\sim 7$ to $z=0$ (Scoville et al. 2017). Galaxies on and near the starforming sequence then grow largely in equilibrium with the supply of gas made available to them (e.g., Davé et al. 2011; Lilly et al. 2013; Peng \& Maiolino 2014).

Massive, quiescent galaxies, in contrast, are generally consistent with passive evolution over many gigayears, with growth mostly influenced by gas-poor minor mergers but relatively little further star formation. Of course, the presentday quiescent population is composed of galaxies that quenched star formation at a variety of redshifts, with a wide variety of evolutionary histories before and after the epoch of quenching. Here, we ask whether the galaxies we have observed at $z \sim 0.7$ are consistent with the population of present-day massive, quiescent galaxies, assuming no external gas accretion further influences their evolution after the epoch at which we observe them.

To address this question, we construct a closed-box toy model, in which a galaxy at $z=0.75$ with $\log M_{\text {star }} / M_{\odot}=11$ continues to deplete its reservoir of molecular gas in a fashion similar to the galaxies we have observed. Namely, we assume a range of initial gas fractions of $f_{\mathrm{H}_{2}}=0.03-0.12$ at $z=0.7$, which decline with time as low-level star formation continues. The evolving (declining) SFRs are determined by assuming a range of depletion times similar to our observations, $t_{\text {dep }}=0.7-1.3 \mathrm{Gyr}$, that remain constant over time. We assume that a long-term average of $\approx 30 \%$ of the mass of stars formed is returned to the ISM through stellar mass loss (Leitner \& Kravtsov 2011; this is also the same assumption made by Scoville et al. 2017 to infer high gas accretion rates for starforming galaxies). Our conclusions are not sensitive to this assumption because the gas masses, and hence SFRs and additional stellar mass formed, are low. The result of this calculation is shown as the densely dashed red line and region in Figure 9, and we compare to nearby quiescent galaxies with $\log M_{\text {star }} / M_{\odot}>10.7$ selected from the $\mathrm{ATLAS}^{3 \mathrm{D}}$ and MASSIVE surveys (Young et al. 2011; Davis et al. 2016).

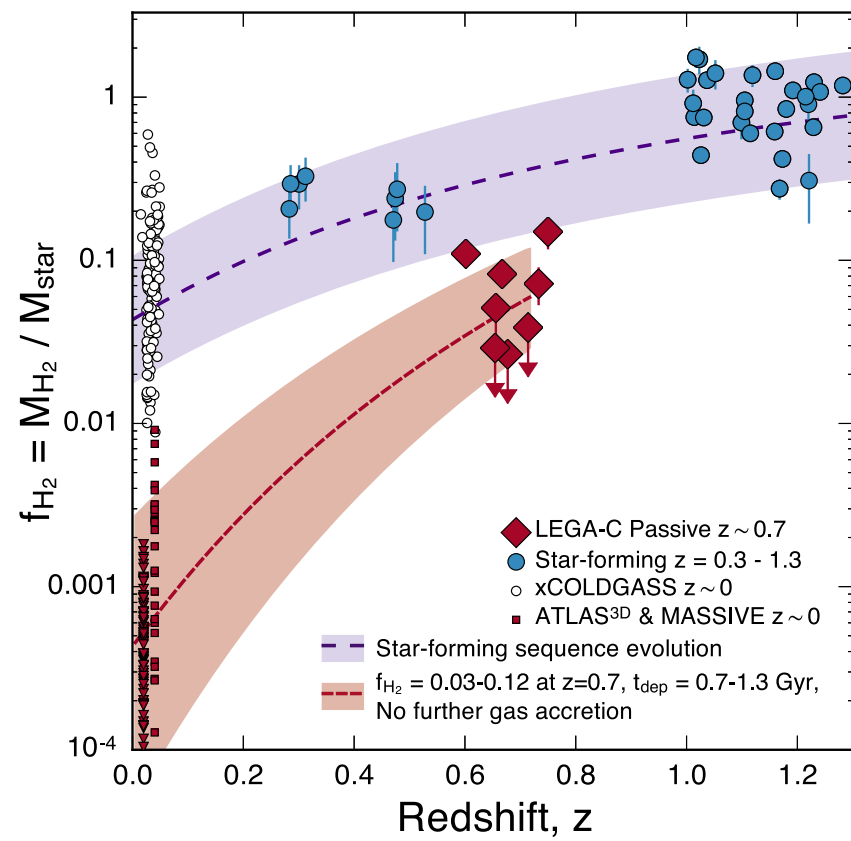

Figure 9. Evolution of $f_{\mathrm{H}_{2}}$ with redshift for galaxies on and below the starforming sequence. Our observations are shown with red diamonds, blue circles show galaxies near the star-forming sequence at $0.3<z<1.3$ (Bauermeister et al. 2013; Tacconi et al. 2013), white circles are $z<0.05$ star-forming galaxies with $10<\log M_{\text {star }} / M_{\odot}<10.7$ from xCOLDGASS (Saintonge et al. 2017), and small red symbols show massive $\left(\log M_{\text {star }} / M_{\odot}>10.7\right)$, quiescent galaxies from the ATLAS ${ }^{3 \mathrm{D}}$ and MASSIVE surveys (Young et al. 2011; Davis et al. 2016). For clarity of presentation, all detections and nondetections from these last two surveys are plotted at $z=0.04$ and $z=0.02$, respectively; in reality, all of these galaxies are within $110 \mathrm{Mpc}(z<0.025)$. The densely dashed red line and region sketch a plausible evolutionary path for the passive galaxies we observed from $z=0.7$ to $z=0$, assuming initial starting $f_{\mathrm{H}_{2}}=0.03-0.12$ and constant $t_{\mathrm{dep}}=0.7-1.3 \mathrm{Gyr}$ and no subsequent accretion of gas. The purple dashed line and region show the evolution for galaxies with $10<\log M_{\text {star }} / M_{\odot}<11$ and within a factor of 3 of the star-forming sequence from Tacconi et al. (2018), where the wide range of these parameters is intended to smooth over the trajectory of individual galaxies around the starforming sequence. The continuity of this relation and the star-forming sequence requires substantial gas accretion over cosmic time. This figure demonstrates that the galaxies we have observed at $z \sim 0.7$ can naturally reproduce the observed gas fractions of the local massive, quiescent population simply by maintaining the same (relatively short) $t_{\mathrm{dep}}$ we measure, with no need for further gas accretion or dynamical stabilization.

We find that a population of intermediate-redshift galaxies with properties similar to our observed sample can very naturally reproduce the range of $f_{\mathrm{H}_{2}}$ seen in nearby passive galaxies, with no need for further gas accretion or dramatic increase in $t_{\mathrm{dep}}$. Alternatively, if additional gas is accreted, it likely remains ionized or neutral, instead of molecular. The same conclusion does not hold for more "normal" star-forming galaxies (blue and white points, and purple dashed line and region in Figure 9), which show gas fractions elevated well above what would be expected in the case of negligible gas accretion. If indeed galaxies similar to those we have observed are progenitors of the local quiescent population, the molecular gas fraction must fall more rapidly with redshift than it does for star-forming galaxies in order to reconcile the observations at $z=0$ and $z \sim 0.7$.

It is also worth noting that the low-level star formation in our toy model does not contradict conclusions about the ages of the stellar populations of quiescent galaxies at $z \sim 0.7$ and lower redshift. Wu et al. (2018) compared the stellar age distributions for quiescent galaxies from LEGA-C at intermediate redshift 
with the ages of similarly massive quiescent galaxies at $z \sim 0.1$ from the Sloan Digital Sky Survey. They found a typical stellar age difference of only $\sim 3 \mathrm{Gyr}$, while the universe aged $\approx 5.5 \mathrm{Gyr}$; in other words, the low-redshift quiescent galaxies are younger than would be expected in the case of purely passive evolution. Wu et al. (2018) surmised that either galaxies with younger stellar ages must join the quiescent population in the intervening time, or that low-level star formation in the existing quiescent galaxies must occur to lower the typical light-weighted stellar age. A true quantitative examination of this point is plagued by degeneracies, but at least qualitatively, the molecular gas fractions and depletion times observed in our sample should improve, rather than worsen, the differences in the stellar ages of quiescent objects at $z \sim 0.7$ and 0.1 .

Our finding that passive galaxies at $z \sim 0.7$ and $z=0$ can be very naturally linked through internal processes continuing to deplete the gas reservoirs provides another independent line of evidence that preventing gas accretion or cooling to molecular form is key to maintaining low SFRs over many gigayears. Several proposed physical mechanisms can accomplish this, including preventing accretion of cold streams or satellite galaxies through the hot circumgalactic medium in massive halos (e.g., Birnboim \& Dekel 2003; Johansson et al. 2009), and radio- or "maintenance-mode" feedback from AGNs to prevent cooling (e.g., Croton et al. 2006). Indeed, Barišić et al. (2017) concluded that radio-mode feedback is likely at work in the most massive galaxies in the LEGA-C sample, acting to maintain the observed low SFRs over several Gyr.

\section{Conclusions and Outlook}

In this work, we have presented ALMA observations of the $\mathrm{CO}(2-1)$ line in a sample of eight massive galaxies at $z \sim 0.7$ selected to lie significantly below the star-forming sequence of galaxies at this redshift, drawn from the LEGA-C spectroscopic survey. Our observations provide a first foray into understanding the molecular ISM properties of passive galaxies outside the local universe. With modest integration by ALMA, we significantly detect $\mathrm{CO}$ emission from four objects at $>6-8 \sigma$, but we do not detect the other four even after stacking, yielding upper limits on the molecular mass $M_{\mathrm{H}_{2}}$. At least three of the four detected galaxies show velocity gradients in the $\mathrm{CO}$ emission typical of rotation, and this rotation appears consistent with the stellar rotation seen in the LEGA-C spectra.

The main focus of this work has been to understand the molecular ISM properties of these passive galaxies in the context of star-forming and quiescent galaxies at $0<z<1.5$. In particular, given the large investment in understanding how gas properties such as $f_{\mathrm{H}_{2}}$ and $t_{\mathrm{dep}}$ vary with other galaxy properties for the star-forming population, we examined whether galaxies farther below the star-forming sequence also follow these scaling relations. We find evidence that passive galaxies at intermediate redshifts have both lower gas fractions and shorter depletion times than expected based on extrapolations from observations of the star-forming population, particularly for the objects undetected in $\mathrm{CO}$ emission. This suggests either a break in the scaling relations at sSFRs $\approx 4-5 \times$ below the star-forming sequence, or a large increase in the scatter of individual galaxy gas properties. On the other hand, a comparison to the EAGLE cosmological simulation indicates that the $\mathrm{CO}$-undetected objects are in reasonable agreement with comparable simulated galaxies, while the CO-detected objects in our sample are somewhat more gas-rich than those seen in the simulation. In both cases, the differences between observations and the predicted scaling relations are likely linked to the physical processes responsible for galaxy quenching, which are not incorporated into the purely empirical observational scaling relations and are uncertain in the simulation.

The generally short depletion times we observe are more consistent with active feedback processes preventing star formation, rather than dynamical or gravitational stabilization against collapse of the existing gas reservoirs. We also show that a population of galaxies like those we have observed can very naturally reproduce the observed gas fractions of $z \sim 0$ early-type galaxies while maintaining the depletion times we observe, with no need for additional gas accretion or increased depletion times predicted by dynamical stabilization. We see no evidence of environmental effects causing the variations in $f_{\text {gas }}$ and $t_{\text {dep }}$ we observe, though we stress that the small sample size of our study makes this inconclusive.

Developing a fuller understanding of the range of gas masses, fractions, and depletion times in high-redshift quiescent galaxies will require observations of a significantly larger sample of objects than we have examined here. This will allow us to determine whether passive galaxies truly show a break toward low $M_{\mathrm{H}_{2}}$ compared to expectations or whether this is merely an artifact of limited sample size. While the dynamical information provided by $\mathrm{CO}$ spectroscopic observations is valuable, it is also clear that a larger sample of passive galaxies will require alternate methods of determining gas masses, such as observing long-wavelength dust continuum emission. Detecting gas fractions at the level of the stacked nondetections in CO with ALMA, for example, would require over an order of magnitude more observing time than we have invested here; reaching the same level in the $870 \mu \mathrm{m}$ dust continuum would require about the same amount of on-source integration as our present observations. Clearly the detection of molecular gas in passive galaxies at high redshift remains a significant challenge, even with the sensitivity of ALMA. Nevertheless, these observations present a new avenue to probe the physics of galaxy quenching and the emergence of the dichotomy between star-forming and quiescent galaxies.

J.S. thanks the McDonald Observatory at the University of Texas at Austin for support through a Harlan J. Smith Fellowship. C.L. is funded by a Discovery Early Career Researcher Award (DE150100618) of the Australian Research Council. C.C.W. acknowledges support from the National Science Foundation Astronomy and Astrophysics Fellowship grant AST-1701546. This paper makes use of the following ALMA data: ADS/JAO.ALMA\#2016.1.00790.S. ALMA is a partnership of ESO (representing its member states), NSF (USA), and NINS (Japan), together with NRC (Canada), NSC and ASIAA (Taiwan), and KASI (Republic of Korea), in cooperation with the Republic of Chile. The Joint ALMA Observatory is operated by ESO, AUI/NRAO, and NAOJ. The National Radio Astronomy Observatory is a facility of the National Science Foundation operated under cooperative agreement by Associated Universities, Inc. This work is based on observations made with ESO Telescopes at the La Silla Paranal Observatory under program ID 194-A.2005 (the LEGA-C Public Spectroscopic Survey).

Facility: ALMA. 


\section{ORCID iDs}

Justin Spilker (i) https://orcid.org/0000-0003-3256-5615 Rachel Bezanson (i) https://orcid.org/0000-0001-5063-8254 Ivana Barišić (iD https://orcid.org/0000-0001-6371-6274 Eric Bell (i) https://orcid.org/0000-0002-5564-9873 Claudia del P. Lagos (iD https://orcid.org/0000-00033021-8564

Michael Maseda (10) https://orcid.org/0000-0003-0695-4414 Adam Muzzin (iD https://orcid.org/0000-0002-9330-9108 Camilla Pacifici (i) https://orcid.org/0000-0003-4196-0617 David Sobral (ii) https://orcid.org/0000-0001-8823-4845 Caroline Straatman (i) https://orcid.org/0000-0001-5937-4590 Arjen van der Wel (i) https://orcid.org/0000-0002-5027-0135 Pieter van Dokkum (iD https://orcid.org/0000-0002-8282-9888 Benjamin Weiner (D) https://orcid.org/0000-0001-6065-7483

Katherine Whitaker (iD https://orcid.org/0000-0001-7160-3632 Christina C. Williams (1) https://orcid.org/0000-00032919-7495

Po-Feng Wu (iD https://orcid.org/0000-0002-9665-0440

\section{References}

Alatalo, K., Davis, T. A., Bureau, M., et al. 2013, MNRAS, 432, 1796 Asayama, S., Takahashi, T., Kubo, K., et al. 2014, PASJ, 66, 57 Barišić, I., van der Wel, A., Bezanson, R., et al. 2017, ApJ, 847, 72 Bauermeister, A., Blitz, L., Bolatto, A., et al. 2013, ApJ, 768, 132 Bezanson, R., van der Wel, A., Pacifici, C., et al. 2018, ApJ, 858, 60 Birnboim, Y., \& Dekel, A. 2003, MNRAS, 345, 349

Bolatto, A. D., Wolfire, M., \& Leroy, A. K. 2013, ARA\&A, 51, 207 Bothwell, M. S., Wagg, J., Cicone, C., et al. 2014, MNRAS, 445, 2599 Chabrier, G. 2003, PASP, 115, 763

Chauke, P., van der Wel, A., Pacifici, C., et al. 2018, ApJ, in press (arXiv:1805. 02568)

Combes, F., Young, L. M., \& Bureau, M. 2007, MNRAS, 377, 1795 Correa, C. A., Schaye, J., Wyithe, J. S. B., et al. 2018, MNRAS, 473, 538 Crain, R. A., Schaye, J., Bower, R. G., et al. 2015, MNRAS, 450, 1937 Croton, D. J., Springel, V., White, S. D. M., et al. 2006, MNRAS, 365, 11 Dannerbauer, H., Daddi, E., Riechers, D. A., et al. 2009, ApJL, 698, L178 Darvish, B., Mobasher, B., Sobral, D., et al. 2016, ApJ, 825, 113 Davé, R., Finlator, K., \& Oppenheimer, B. D. 2011, MNRAS, 416, 1354 Davé, R., Finlator, K., \& Oppenheimer, B. D. 2012, MNRAS, 421, 98 Davis, T. A., Alatalo, K., Bureau, M., et al. 2013, MNRAS, 429, 534 Davis, T. A., Greene, J., Ma, C.-P., et al. 2016, MNRAS, 455, 214 Draine, B. T., \& Li, A. 2007, ApJ, 657, 810

Driver, S. P., Andrews, S. K., da Cunha, E., et al. 2018, MNRAS, 475, 2891 Dunne, L., Eales, S., Edmunds, M., et al. 2000, MNRAS, 315, 115 Fabian, A. C. 2012, ARA\&A, 50, 455

Fixsen, D. J., Bennett, C. L., \& Mather, J. C. 1999, ApJ, 526, 207

Förster Schreiber, N. M., Genzel, R., Bouché, N., et al. 2009, ApJ, 706, 1364 Franx, M., van Dokkum, P. G., Förster Schreiber, N. M., et al. 2008, ApJ, 688,770

Fumagalli, M., Labbé, I., Patel, S. G., et al. 2014, ApJ, 796, 35 Furlong, M., Bower, R. G., Theuns, T., et al. 2015, MNRAS, 450, 4486 Gallazzi, A., Bell, E. F., Zibetti, S., Brinchmann, J., \& Kelson, D. D. 2014, ApJ, 788, 72

Gavazzi, G., Consolandi, G., Dotti, M., et al. 2015, A\&A, 580, A116

Geach, J. E., Chapin, E. L., Coppin, K. E. K., et al. 2013, MNRAS, 432, 53 Genzel, R., Förster Schreiber, N. M., Lang, P., et al. 2014, ApJ, 785, 75

Genzel, R., Tacconi, L. J., Lutz, D., et al. 2015, ApJ, 800, 20

Gnedin, N. Y., \& Kravtsov, A. V. 2011, ApJ, 728, 88

Gobat, R., Daddi, E., Magdis, G., et al. 2018, NatAs, 2, 239

Hayward, C. C., Lanz, L., Ashby, M. L. N., et al. 2014, MNRAS, 445, 1598 Johansson, P. H., Naab, T., \& Ostriker, J. P. 2009, ApJL, 697, L38

Kauffmann, G., Heckman, T. M., White, S. D. M., et al. 2003, MNRAS, 341,33
Kennicutt, R. C., Jr. 1998, ApJ, 498, 541

Kereš, D., Katz, N., Weinberg, D. H., \& Davé, R. 2005, MNRAS, 363, 2

Kriek, M., van Dokkum, P. G., Labbé, I., et al. 2009, ApJ, 700, 221

Krumholz, M. R. 2013, MNRAS, 436, 2747

Lagos, C. d. P., Crain, R. A., Schaye, J., et al. 2015, MNRAS, 452, 3815

Lagos, C. d. P., Stevens, A. R. H., Bower, R. G., et al. 2018, MNRAS, 473, 4956

Lagos, C. d. P., Theuns, T., Schaye, J., et al. 2016, MNRAS, 459, 2632

Leitner, S. N., \& Kravtsov, A. V. 2011, ApJ, 734, 48

Leroy, A. K., Bolatto, A., Gordon, K., et al. 2011, ApJ, 737, 12

Lilly, S. J., Carollo, C. M., Pipino, A., Renzini, A., \& Peng, Y. 2013, ApJ, 772,119

Madau, P., \& Dickinson, M. 2014, ARA\&A, 52, 415

Magnelli, B., Lutz, D., Saintonge, A., et al. 2014, A\&A, 561, A86 Maltby, D. T., Almaini, O., Wild, V., et al. 2016, MNRAS, 459, L114

Man, A. W. S., Greve, T. R., Toft, S., et al. 2016, ApJ, 820, 11

Martig, M., Bournaud, F., Teyssier, R., \& Dekel, A. 2009, ApJ, 707, 250

Martig, M., Crocker, A. F., Bournaud, F., et al. 2013, MNRAS, 432, 1914

McAlpine, S., Helly, J. C., Schaller, M., et al. 2016, A\&C, 15, 72

Muzzin, A., Marchesini, D., Stefanon, M., et al. 2013, ApJS, 206, 8

Narayanan, D., Krumholz, M. R., Ostriker, E. C., \& Hernquist, L. 2012, MNRAS, 421, 3127

Noeske, K. G., Weiner, B. J., Faber, S. M., et al. 2007, ApJL, 660, L43

Papovich, C., Labbé, I., Glazebrook, K., et al. 2016, NatAs, 1, 0003

Peng, Y.-j., Lilly, S. J., Kovač, K., et al. 2010, ApJ, 721, 193

Peng, Y.-j., \& Maiolino, R. 2014, MNRAS, 443, 3643

Planck Collaboration, Ade, P. A. R., Aghanim, N., et al. 2016, A\&A, 594, A13

Popping, G., Decarli, R., Man, A. W. S., et al. 2017, A\&A, 602, A11 Saintonge, A., Catinella, B., Tacconi, L. J., et al. 2017, ApJS, 233, 22

Saintonge, A., Kauffmann, G., Kramer, C., et al. 2011, MNRAS, 415, 32

Salim, S., Dickinson, M., Michael Rich, R., et al. 2009, ApJ, 700, 161

Sandstrom, K. M., Leroy, A. K., Walter, F., et al. 2013, ApJ, 777, 5

Sargent, M. T., Daddi, E., Bournaud, F., et al. 2015, ApJL, 806, L20

Schaye, J., Crain, R. A., Bower, R. G., et al. 2015, MNRAS, 446, 521

Schmidt, M. 1959, ApJ, 129, 243

Schreiber, C., Elbaz, D., Pannella, M., et al. 2016, A\&A, 589, A35

Schreiber, C., Pannella, M., Elbaz, D., et al. 2015, A\&A, 575, A74

Schruba, A., Leroy, A. K., Walter, F., et al. 2011, AJ, 142, 37

Scoville, N., Lee, N., Vanden Bout, P., et al. 2017, ApJ, 837, 150

Scoville, N., Sheth, K., Aussel, H., et al. 2016, ApJ, 820, 83

Sell, P. H., Tremonti, C. A., Hickox, R. C., et al. 2014, MNRAS, 441, 3417

Smolčić, V., Novak, M., Bondi, M., et al. 2017, A\&A, 602, A1

Sobral, D., Best, P. N., Smail, I., et al. 2011, MNRAS, 411, 675

Sobral, D., Best, P. N., Smail, I., et al. 2014, MNRAS, 437, 3516

Solomon, P. M., Rivolo, A. R., Barrett, J., \& Yahil, A. 1987, ApJ, 319, 730

Sparre, M., Hayward, C. C., Springel, V., et al. 2015, MNRAS, 447, 3548

Speagle, J. S., Steinhardt, C. L., Capak, P. L., \& Silverman, J. D. 2014, ApJS, 214, 15

Spilker, J. S., Aravena, M., Marrone, D. P., et al. 2015, ApJ, 811, 124

Spilker, J. S., Bezanson, R., Marrone, D. P., et al. 2016, ApJ, 832, 19

Spilker, J. S., Marrone, D. P., Aguirre, J. E., et al. 2014, ApJ, 785, 149

Suess, K. A., Bezanson, R., Spilker, J. S., et al. 2017, ApJL, 846, L14

Tacconi, L. J., Genzel, R., Saintonge, A., et al. 2018, ApJ, 853, 179

Tacconi, L. J., Neri, R., Genzel, R., et al. 2013, ApJ, 768, 74

Tomczak, A. R., Quadri, R. F., Tran, K.-V. H., et al. 2016, ApJ, 817, 118

Tremonti, C. A., Moustakas, J., \& Diamond-Stanic, A. M. 2007, ApJL, 663, L77

Utomo, D., Kriek, M., Labbé, I., Conroy, C., \& Fumagalli, M. 2014, ApJL, 783, L30

van der Wel, A., Franx, M., van Dokkum, P. G., et al. 2014, ApJ, 788, 28 van der Wel, A., Noeske, K., Bezanson, R., et al. 2016, ApJS, 223, 29 Vogelsberger, M., Genel, S., Springel, V., et al. 2014, MNRAS, 444, 1518 Whitaker, K. E., Franx, M., Leja, J., et al. 2014, ApJ, 795, 104

Whitaker, K. E., van Dokkum, P. G., Brammer, G., \& Franx, M. 2012, ApJL, 754, L29

Wu, P.-F., van der Wel, A., Gallazzi, A., et al. 2018, ApJ, 855, 85

Wuyts, S., Förster Schreiber, N. M., Wisnioski, E., et al. 2016, ApJ, 831, 149

Young, L. M., Bureau, M., Davis, T. A., et al. 2011, MNRAS, 414, 940 